\title{
The Conserved Dopaminergic Diencephalospinal Tract Mediates Vertebrate Locomotor Development in Zebrafish Larvae
}

\author{
Aaron M. Lambert, ${ }^{1}$ Joshua L. Bonkowsky, ${ }^{2,3}$ and Mark A. Masino ${ }^{1}$ \\ ${ }^{1}$ Department of Neuroscience, University of Minnesota, Minneapolis, Minnesota 55455, 2Department of Neurobiology and Anatomy, University of Utah, \\ Salt Lake City, Utah 84132, and ' ${ }^{3}$ Department of Pediatric Neurology and Pediatrics, University of Utah Medical Center, Salt Lake City, Utah 84132
}

The most conserved part of the vertebrate dopaminergic system is the orthopedia (otp)-expressing diencephalic neuronal population that constitutes the dopaminergic diencephalospinal tract (DDT). Although studies in the neonatal murine spinal cord in vitro suggest an early locomotor role of the DDT, the function of the DDT in developing vertebrates in vivo remains unknown. Here, we investigated the role of the DDT in the locomotor development of zebrafish larvae. To assess the development of the behavioral and neural locomotor pattern, we used high-throughput video tracking in combination with peripheral nerve recordings. We found a behavioral and neural correspondence in the developmental switch from an immature to mature locomotor pattern. Blocking endogenous dopamine receptor $4\left(\mathrm{D}_{4} \mathrm{R}\right)$ signaling in vivo either before or after the developmental switch prevented or reversed the switch, respectively. Spinal transections of post-switch larvae reestablished the immature locomotor pattern, which was rescued to a mature-like pattern via spinal $\mathrm{D}_{4} \mathrm{R}$ agonism. Selective chemogenetic ablation of otp $b(o t p b)$ neurons that contribute to the DDT perpetuated the immature locomotor pattern in vivo. This phenotype was recapitulated by diencephalic transections that removed the dopaminergic ot $p b$ population and was rescued to a mature-like locomotor pattern by $\mathrm{D}_{4} \mathrm{R}$ agonism. We conclude that the dopaminergic otp $b$ population, via the DDT, is responsible for spinal $\mathrm{D}_{4} \mathrm{R}$ signaling to mediate the developmental switch to the mature locomotor pattern of zebrafish. These results, integrated with the mammalian literature, suggest that the DDT represents an evolutionarily conserved neuromodulatory system that is necessary for normal vertebrate locomotor development.

\section{Introduction}

Noteworthy similarities and differences are present among the dopaminergic (DAergic) systems across vertebrates. Although midbrain DAergic neurons appear to be a more recent evolutionary development (Reiner et al., 1998; Smeets and Gonzalez, 2000), the DAergic diencephalospinal tract (DDT), which provides the exclusive source of spinal dopamine (DA) in most vertebrates, is highly conserved and may represent an ancient neuromodulatory system of functional relevance to both zebrafish and mammals (Tay et al., 2011). The diencephalic DAergic neurons that comprise the DDT have a conserved somatic location, ascending and descending projections (Takada et al., 1988; Qu et al., 2006; Kastenhuber et al., 2010), and require the

\footnotetext{
Received April 3, 2012; revised July 5, 2012; accepted July 26, 2012.

Author contributions: A.M.L. and M.A.M. designed research; A.M.L. and J.L.B. performed research; J.L.B. contributed unpublished reagents/analytic tools; A.M.L., J.L.B., and M.A.M. analyzed data; A.M.L. and M.A.M. wrote the paper.

This work was supported by National Institutes of Health Translation Research in Neurobiology of Disease Training Grant T32-DA022616 (A.M.L.), National Institutes of Health Grants K08 DA024753 (J.L.B.) and R01-NS065054 (M.A.M.), a Primary Children's Medical Center Foundation grant (J.L.B.), Minnesota Medical Foundation Grant 40529238-11 (M.A.M.), and Office of the Dean of the Graduate School of the University of Minnesota (Grant-in-Aid of Research, Artistry, and Scholarship 21934; M.A.M.). We thank Drs. Teresa Nick and Martha Flanders for helpful comments on this manuscript and John Eian for writing MATLAB code for analysis.

The authors declare no competing financial interests.

Correspondence should be addressed to Mark A. Masino, Department of Neuroscience, University of Minnesota, 321 Church Street, Minneapolis, MN 55455. E-mail: masino@umn.edu.

DOI:10.1523/JNEUROSCI.1638-12.2012

Copyright $\odot 2012$ the authors $\quad 0270-6474 / 12 / 3213488-13 \$ 15.00 / 0$
}

transcription factor orthopedia (otp) for the development of cellular identity in both zebrafish and mammals (Ryu et al., 2007). Although the function of the zebrafish DDT is unknown, the adult mammalian DDT is implicated in human restless leg syndrome (Paulus et al., 2007; Qu et al., 2007; Zhao et al., 2007) and exerts direct antinociceptive actions in the rat spinal cord in vivo (Taniguchi et al., 2011). Conversely, the function of the DDT in developing vertebrates in vivo is unknown, but in vitro and anatomical evidence suggests that the DDT may serve an early role in locomotor development (McEwen et al., 1997; Zhu et al., 2007; Tay et al., 2011).

Zebrafish exhibit a locomotor pattern of episodic beat-and-glide swimming, which consists of discrete swimming episodes interposed with passive gliding. A developmental switch in the episodic locomotor pattern from long to short swimming episodes is observed between 3 and 4 days postfertilization (dpf) (Buss and Drapeau, 2001), which is concurrent with increased locomotion and the advent of foraging (Borla et al., 2002). Interestingly, the DDT does not yet exert a locomotor effect in the spinal cord at $3 \mathrm{dpf}$ (Thirumalai and Cline, 2008), although, at this stage, the otp cells that comprise the DDT are positive for tyrosine hydroxylase $(\mathrm{TH})$ and DA transporter mRNA (dat; slc6a3) and send robust projections across the entire rostrocaudal extent of the spinal cord (McLean and Fetcho, 2004b; Kastenhuber et al., 2010; Fujimoto et al., 2011) that, by $4 \mathrm{dpf}$, form putative synapses with spinal motor neurons (McLean and Fetcho, 2004a). 
We postulated that the DDT becomes functional in the zebrafish spinal cord at $4 \mathrm{dpf}$ to confer the mature locomotor pattern of short swimming episodes that is observed into adulthood (Fuiman and Webb, 1988). To assay the development of the episodic locomotor pattern, the present study introduces a novel adaptation of a high-throughput video-tracking algorithm (Ctrax; Branson et al., 2009) to compare the free-swimming locomotor pattern with peripheral nerve recordings of the neural locomotor output of the spinal cord (Masino and Fetcho, 2005). Through pharmacological perturbations, demarcated transections, and selective chemogenetic ablation of otp neurons, we demonstrate that the conserved DAergic otp neurons that comprise the DDT provide the impetus for spinal DA receptor 4 $\left(\mathrm{D}_{4} \mathrm{R}\right)$ signaling to mediate the developmental switch to the mature episodic locomotor pattern. These findings suggest that the DDT may serve an evolutionarily conserved function in mediating vertebrate locomotor development.

\section{Materials and Methods}

Animals. All experiments were performed on zebrafish (Danio rerio) larvae between 1 and 7 dpf. Wild-type (WT) larvae were obtained from a laboratory stock (Segrest) of adults at the University of Minnesota. Embryos and larvae were raised in an incubator at $28.5^{\circ} \mathrm{C}$ under a $14 / 10$ light/dark cycle (Lights On, 8:00 A.M.; Lights Off, 10:00 P.M.) until the start of behavioral or neural recordings between 3 and $7 \mathrm{dpf}$. Larvae were kept at room temperature during all manipulations and recording sessions and returned to the incubator during interims.

The $\operatorname{Tg}(o t p b . A: n f s B-e g f p)^{\mathrm{zc} 77}$ line was generated at the University of Utah. In the $\operatorname{Tg}\left(o t p b . A: n f_{s} B-e g f p\right)^{\mathrm{zc} 77}$ line, the $o t p b . A$ promoter drives expression of the $n f s B$ enzyme nitroreductase (Ntr). Specific plasmids used for cloning were as follows: p5E-otpb.A (Fujimoto et al., 2011), pME- $n s f B$ (no stop codon) (kind gift from C. Seiler, University of Pennsylvania School of Medicine, Philadelphia, PA), p3E-EGFPpA, and pDestTol2pA2 (Kwan et al., 2007). Injection of DNA constructs and husbandry of stable transgenic lines were performed as described previously (Bonkowsky et al., 2008). All procedures were approved by the Institutional Animal Care and Use Committees at the University of Minnesota and the University of Utah.

Immunohistochemistry, in situ hybridization, and terminal deoxynucleotidyl transferase dUTP nick-end labeling staining. Whole-mount immunohistochemistry was performed as described previously (Bonkowsky et al., 2008; Fujimoto et al., 2011). Embryos were raised in phenylthiourea $(0.003 \%)$ in E3 media to inhibit pigment formation beginning at 24 hours postfertilization (hpf). Antibodies used included the following: rabbit polyclonal anti-TH at 1:400 (Millipore), mouse monoclonal anti-GFP at 1:250 (Millipore), Cy3 anti-rabbit at 1:400 (Invitrogen), and Alexa Fluor 488 donkey anti-mouse at 1:400 (Invitrogen). Whole-mount in situ labeling for dat (Holzschuh et al., 2001) was performed as described previously (Bonkowsky and Chien, 2005; Fujimoto et al., 2011).

Terminal deoxynucleotidyl transferase dUTP nick-end labeling (TUNEL) was performed on whole-mount larvae (ApopTag Fluorescein In Situ Apoptosis Detection Kit; Millipore Bioscience Research Reagents). After standard fixation and dehydration of larvae in $100 \%$ methanol, larvae were rehydrated stepwise into PBS with $0.1 \%$ Tween 20 (PBST), permeabilized with $10 \mathrm{mg} / \mathrm{ml}$ Proteinase $\mathrm{K}$ in PBST at $28^{\circ} \mathrm{C}$, washed twice with PBST, refixed for 20 min with $4 \%$ paraformaldehyde (PFA), and washed with PBST. Subsequently, $75 \mu$ l of equilibration buffer was added to the larvae for $1 \mathrm{~h}$ and then removed and replaced with 55 $\mu l$ of "working-strength" (per Apoptosis Detection Kit instructions) terminal deoxynucleotidyl transferase enzyme overnight at $37^{\circ} \mathrm{C}$. To avoid drying out the larvae, Eppendorf tubes were sealed with Parafilm. Before use, the anti-digoxigenin conjugate was warmed to room temperature. The end-labeling reaction was stopped by washing the embryos three times for $15 \mathrm{~min}$ each with $2 \mathrm{ml}$ of the stop/wash buffer, followed by three 1 min washes with PBS. Then $65 \mathrm{ml}$ of working-strength sheep antidigoxigenin rhodamine was added to the embryos overnight at $4^{\circ} \mathrm{C}$.
Double immunohistochemistry for GFP and TUNEL was performed by TUNEL staining; after washes with PBST, larvae were fixed for $20 \mathrm{~min}$ in $4 \%$ PFA at room temperature and washed again with PBST. Larvae were then permeabilized and antibody stained per our routine protocol (Bonkowsky et al., 2008; Fujimoto et al., 2011).

Microscopy and image analysis. Image acquisition and analysis were performed essentially as described previously (Fujimoto et al., 2011). Images of embryos/larvae processed for in situ or immunohistochemistry were taken using a confocal microscope or bright-field microscope. Embryos/larvae were taken stepwise into a solution of $80 \%$ glycerol $/ 20 \%$ PBST and then mounted on a glass slide with a \#0 coverslip fixed into place over a well made using electrical tape. NIH ImageJ software was used for image processing.

Metronidazole treatments. $\operatorname{Tg}\left(o t p b . A: n f_{s} B-e g f p\right)^{z c 77}$ embryos were dechorionated manually at $24 \mathrm{hpf}$ and placed in $5 \mathrm{~mm}$ metronidazole $(\mathrm{Mtz})$ (Vetranal from Sigma) dissolved in embryo media (0.03\% Instant Ocean in $\mathrm{dH}_{2} \mathrm{O}$ ). These larvae were then transferred to fresh $10 \mathrm{~mm} \mathrm{Mtz}$ and incubated from 48 to $96 \mathrm{hpf}$, after which Mtz was removed through a series of washes in embryo media. Untreated siblings, also dechorionated at $24 \mathrm{hpf}$, remained in embryo media continuously, with fresh media being given at 24, 48, and $96 \mathrm{hpf}$. After Mtz washout at $96 \mathrm{hpf}$ in Mtztreated larvae, untreated and Mtz-treated larvae were given $1 \mathrm{~d}$ in embryo media before behavioral and neural locomotor activity was assessed at 5 dpf. Genotypes for untreated and Mtz-treated larvae were screened as Ntr-negative $\left[\mathrm{Ntr}^{-}\left(\right.\right.$egfp $\left.\left.{ }^{-}\right)\right]$or Ntr-positive $\left[\mathrm{Ntr}^{+}\left(\operatorname{egfp}^{+}\right)\right]$via an epifluorescent stereoscope (MZ16FA; Leica). Only larvae that exhibited an inflated swim bladder at $5 \mathrm{dpf}$ were included in analyses for all experimental groups.

Pharmacology. All drug concentrations were determined in preliminary concentration-response experiments (data not shown). For each pharmacologic agent, we determined the lowest drug concentration that achieved a maximal locomotor effect rather than the $\mathrm{IC}_{50}$ or $\mathrm{EC}_{50}$ concentration that would only induce a half-maximal effect. As such, the lowest concentration for each drug that achieved a maximal locomotor effect was subsequently used for the reported experiments. For each DA receptor (DAR)-specific antagonist, for example, this concentration was determined to be $10 \mu \mathrm{M}$, which is consistent with the range of efficacious concentrations determined during high-throughput behavioral screening of $5280(10-30 \mu \mathrm{m}$; Rihel et al., 2010) and 14,000 (10-100 $\mu \mathrm{M}$; Kokel et al., 2010) compounds in zebrafish larvae and embryos, respectively. NMDA, dimethylsulfoxide (DMSO), DA, Mtz, DAR-specific antagonists $\left(\mathrm{D}_{1} \mathrm{R}, \mathrm{SCH}-23390[R(+)-7-\right.$ chloro-8-hydroxy-3-methyl-1-phenyl-2,3,4,5-tetrahydro-1H-3-benzazepine hydrochloride]; $\mathrm{D}_{2} / \mathrm{D}_{3} \mathrm{R}$, raclopride [3,5-Dichloro- $\mathrm{N}$-(1-ethylpyrrolidin-2-ylmethyl)-2-hydroxy-6-methoxybenzamide]; $\mathrm{D}_{4} \mathrm{R}, \mathrm{L}-745,870$ (3[[4-(4-chlorophenyl)piperazin-1-yl] methyl]-1 H-pyrrolo[2,3-b]pyridine trihydrochloride)), and DAR-specific agonists $\left(\mathrm{D}_{1}\right.$-like, SKF-38393 [2,3,4,5tetrahydro-7,8-dihydroxy-1-phenyl-1 $\mathrm{H}$-3-benazepine $\mathrm{HCl}$ ]; $\mathrm{D}_{2}$-like, quinpirole (trans-( -)-(4aR)-4,4a,5,6,7,8,8a,9-Octahydro-5-propyl-1Hpyrazolo[3,4-g]); $\mathrm{D}_{4} \mathrm{R}, \mathrm{PD} 168,077$ [N-[[4-(2-cyanophenyl)piperazin-1yl]methyl]-3-methylbenzamide)] were obtained from Sigma.

Chronic drug incubations. For chronic incubations of DAR-specific antagonists to WT larvae, drugs were dissolved at stated concentrations in embryo media containing $0.1 \%$ DMSO; low concentrations of DMSO facilitate in aqueous drug delivery through the skin without affecting behavior (Rihel et al., 2010). Larvae subjected to embryo media containing $0.1 \%$ DMSO served as vehicle controls. Baseline free-swimming behavior of all groups was video recorded before the start of drug incubations and comprises the control data reported for 3 and $4 \mathrm{dpf}$ larvae. Chronic drug incubations began at 7:00 P.M. for behavioral and neural assessments on the following day. All experimental groups remained in their respective drugs throughout the recording day, which spanned from 9:00 AM through 6:00 P.M. During all behavioral recording sessions, experimental groups remained in their respective drugs. For neural recording sessions of pilot experiments, preparations were superfused in their respective drugs. It was determined that the effect of chronic drug incubations did not wash out after removal of the drug via superfusion in extracellular solution alone (see below, Electrophysiology) during the $\sim 30$ min recording session. As such, neural recording sessions occurred in the presence of extracellular solution without drug. For each experi- 
mental condition, behavioral and neural recordings were each performed on a minimum of three different recording days, coming from a minimum of three different clutches. Behavioral and neural recordings for each experimental condition were equally sampled across the recording day and were pooled regardless of recording time.

Video acquisition. A group of 10 larvae in embryo media or embryo media containing drug were transferred to a $50 \mathrm{~mm}$ watch glass (Thermo Fisher Scientific) positioned atop a transmitted light stage (Schott TLS and MC-1500 LED Controller). The light intensity at the level of the arena was set to 15,000 lux to maximize contrast and facilitate tracking of dark targets on a light background. The aqueous depth was $10 \mathrm{~mm}$ at the center of the arena and became progressively shallower toward the periphery. The larvae acclimated to the recording arena for $5 \mathrm{~min}$ before the start of video recording. Spontaneous free-swimming was recorded for $10 \mathrm{~min}$ at $60 \mathrm{frames} / \mathrm{s}$ with a digital CMOS camera (Firefly MV; Point Gray Research) with an attached $50 \mathrm{~mm}$ macro lens (Sigma). Videos were acquired, via Fview (open-source software; Straw and Dickinson, 2009), in uncompressed fly movie format (FMF).

Video analysis. The FMF files generated by Fview were imported into the California Institute of Technology Fly Tracker (Ctrax) (open-source software; Branson et al., 2009) to obtain independent trajectories of each target within the arena. We used the open-source Fix Errors MATLAB Toolbox (FEMT), provided by the creators of Ctrax (Branson et al., 2009), to identify and fix tracking errors (such as swapping of target identities). The total number of errors per 10-minute video was $3.89 \pm$ $0.38(n=100$ videos), and all errors were corrected via the FEMT.

Subsequently, scripts from the open-source Behavioral Microarray MATLAB Toolbox, provided by the creators of Ctrax (Branson et al., 2009), were implemented to compute descriptive statistics of a suite of behavioral parameters for each of the individual targets from the fixed Ctrax trajectories. The target speed function, velmag, was extracted for each target to define and detect event onsets and offsets by thresholding the speed function at $1.5 \mathrm{~mm} / \mathrm{s}$. This threshold was sufficiently above most baseline noise, which was, on average, $<0.15 \mathrm{~mm} / \mathrm{s}$. To filter out transient high-amplitude noise (i.e., $>1.5$ and $<3.0 \mathrm{~mm} / \mathrm{s}$ ), only events that spanned at least four consecutive frames with an average speed of 3 $\mathrm{mm} / \mathrm{s}$ or greater were classified as swimming episodes. Finally, $3 \mathrm{dpf}$-like swimming consisted of long episodes characterized by continuous active undulations when viewing the video frame by frame, but larvae sometimes exhibited periods of the active swimming episode with little or no displacement. Consequently, such periods during an active swimming episode are not captured in the unfiltered speed function. To better capture the entire continuous swimming episode, events that occurred within an 18 frame $(300 \mathrm{~ms})$ interevent interval were concatenated into a single swimming episode. This classification system did not erroneously concatenate multiple individual swimming episodes because the shortest latencies between successive free-swimming episodes of zebrafish larvae between 3 and $9 \mathrm{dpf}$ exceed $300 \mathrm{~ms}$ and on average exceed $800 \mathrm{~ms}$ (Fuiman and Webb, 1988; Müller et al., 2000; Farrell et al., 2011). Identical filters were applied for all videos across all ages and experimental groups.

Once all swimming episodes were identified, we quantified the total distance traveled per individual in centimeters (sum of instantaneous speeds during swimming episodes divided by the frame rate) and mean swimming episode duration per individual in milliseconds (mean duration from the onset to the offset of each individual episode) over the 10 -minute recording period. Sample sizes were ultimately smaller for quantifying episode duration compared with total distance traveled because episode duration could not be obtained from animals that did not move during the recording session. Values for total distance traveled and episode duration are expressed as mean \pm SD.

Electrophysiology. Neural recordings from paralyzed preparations (i.e., fictive swimming) were assessed via peripheral nerve recordings in vivo as described previously (Masino and Fetcho, 2005). Larvae were anesthetized with $0.02 \%$ Tricaine-S (Western Chemical) in extracellular recording solution that contained the following (in $\mathrm{mm}$ ): $134 \mathrm{NaCl}, 3 \mathrm{KCl}, 1.2$ $\mathrm{MgCl}_{2}, 2.1 \mathrm{CaCl}_{2}, 10 \mathrm{HEPES}$ buffer, and 10 glucose, adjusted to $\mathrm{pH} 7.8$ with $\mathrm{NaOH}$ (osmolarity adjusted to $290 \mathrm{mOsm}$ with sucrose). Larvae were transferred to Sylgard-lined dissecting dishes and pinned on their sides through the notochord using short pieces of fine tungsten wire (0.001 inch diameter). To access the peripheral motor nerves, the skin was removed between the tungsten pins using a sharp tungsten probe and fine forceps (Fine Science Tools). To prevent muscle contractions during recording, larvae were paralyzed using $5 \mu \mathrm{l}$ of $0.1 \mathrm{~mm} \alpha$-bungarotoxin (Tocris Bioscience) added to the small amount $(\sim 15 \mu \mathrm{l})$ of extracellular solution in the dissection dish. Subsequently, larvae were transferred to the stage of an Olympus BX51 WI microscope, and extracellular recording solution was superfused at room temperature. Suction electrodes (6-15 $\mu \mathrm{m}$ tip diameter), filled with extracellular recording solution, were placed in an electrode holder and positioned over the peripheral nerves using micromanipulators (Siskiyou). All recordings were between midbody segments 10 and 20 and were acquired using a MultiClamp 700B amplifier (Molecular Devices), a Digidata series 1440A digitizer (Molecular Devices), and pClamp 10.2 software (Molecular Devices). Extracellular voltage was monitored in current-clamp mode at a gain of $2000\left(R_{\mathrm{f}}=50 \mathrm{M} \Omega\right)$ with the low- and high-frequency cutoff at 300 and $1000 \mathrm{~Hz}$, respectively.

For all preparations in which spontaneous fictive swimming was assessed, spontaneous fictive activity was recorded for a minimum of 30 min. For acute drug administration $(\sim 30 \mathrm{~min})$ to fictive preparations, drugs were dissolved at stated concentrations in extracellular recording solution and superfused at $1 \mathrm{ml} / \mathrm{min}$. NMDA $(50 \mu \mathrm{M})$ was superfused for 15-20 min to elicit a baseline of NMDA-induced fictive swimming before application of DA or DAR agonists. DA or DAR agonists were superfused with NMDA for 10-15 min before returning to an NMDA solution (washout).

Analysis of peripheral nerve activity. A program written in MATLAB (MathWorks) was used to analyze extracellular peripheral nerve voltage recordings. The program detected the presence or absence of activity at each voltage sample $[v(n)]$. For each $v(n)$, the algorithm determined a voltage autocorrelation $\left[c_{n}(k)\right]$ over a small window $(3 \mathrm{~ms})$ centered at $v(n)$. These "windowed" autocorrelations were computed as follows:

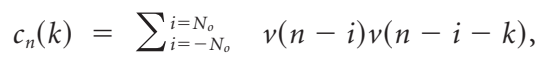

where $3 \mathrm{~ms}$ windowing was implemented by setting $N_{0}=(3 \mathrm{~ms} \times$ $\left.f_{\text {sam }}\right) / 2$, where $f_{\text {sam }}$ is the sampling frequency, and by setting $v(j)=0$ for $j$ outside the interval $\left[n-N_{0}, n+N_{0}\right]$.

A subset of the autocorrelation values (lags) from Equation 1 were used to compute a test statistic for each $v(n)$ with the same lags $\left(\vec{k}_{o}=\left[k_{1}, k_{2}, \ldots, k_{m}\right]\right)$ used for all voltage samples. Building on Equation 1, for each $v(n)$, a test statistic $c_{n}$ was computed as follows:

$$
c_{n}=\sum_{k=k_{1}}^{k=k_{m}} \sum_{\substack{i=N_{o} \\ i=N_{o}}} v(n-i) v(n-i-k),
$$

where Equation 2 is the sum of the $c_{n}(k)$ from Equation 1 specified by $\vec{k}_{\mathrm{o}}$. $\vec{k}_{\mathrm{o}}$ was set at $\vec{k}_{\mathrm{o}}=[1,2]$ to separate the distributions of the test statistics $\left\{c_{n}\right\}$ in the cases of noise versus activity.

Finally, activity was considered present at $v(n)$ only when $c_{n}$ was greater than a detection threshold $T$. $T$ was set as the maximum of a set of $\left\{c_{n}\right\}$ corresponding to the $\{v(n)\}$ in 1 contiguous second of the voltage recording in which activity was absent (typically the first second of the recording) and was set this way for each individual voltage recording to account for differences in gain settings and/or baseline noise levels. Fictive locomotor bursts were detected, grouped into episodes, and the burst and episode properties (duration, frequency, and interburst or inter-episode intervals) for each voltage trace were determined as described previously (Masino and Fetcho, 2005). Values for episode duration are expressed as mean $\pm \mathrm{SD}$.

Transections of the nervous system. Transections at various levels of the nervous system were made with a fine razorblade (FA-10 Feather S; Ted Pella) held with a blade breaker and holder (10053-09; Fine Science Tools). Larvae were anesthetized and prepared for electrophysiological recordings as described above. Spinalized preparations were generated in both WT and $\operatorname{Tg}(o t p b . A: n f s B-e g f p)^{\text {zc77 }}$ larvae between 4 and 7 dpf by transecting the nervous system between body segments 3 and 4 , just caudal to the hindbrain-spinal cord junction (between body segments 2 and 3). Spinalization completely separated the brain from the spinal 
cord, ensuring that all descending inputs to the transected spinal cord were eliminated.

To target mid-diencephalic and caudal-diencephalic transections, $\operatorname{Tg}(o t p b . A: n f s B-e g f p)^{\mathrm{zc} 77}$ larvae between 4 and $7 \mathrm{dpf}$ were used to visualize egfp ${ }^{+}$tp $b$ cell populations using an epifluorescent stereoscope (MZ16FA; Leica). Following preparation for electrophysiological recordings as described above, larvae were reoriented dorsoventrally to visualize egfp ${ }^{+}$otpb populations. Mid-diencephalic transections were made between rostral, non-DAergic and caudal, DAergic diencephalic $o t p b$ populations. Caudal diencephalic transections were made just posterior to the caudal DAergic diencephalic otpb population. Confirmation of successful transections was visually verified by the presence of only the egfp ${ }^{+}$DAergic $o t p b$ population in mid-diencephalic-transected (MDT) preparations and the absence of all egfp ${ }^{+}$otp $b$ populations in caudaldiencephalic-transected (CDT) preparations.

Statistical analyses. Statistical analyses were performed with SigmaPlot 11.0 (Systat Software). Data were analyzed using Student's $t$ tests (two conditions only), one-way ANOVAs (more than two conditions), or two-way ANOVAs [first factor: condition (either age or drug exposure) assayed by two different locomotor outputs (second factor: free swimming or fictive swimming)]. These statistical tests accounted for repeated measures when appropriate, namely when comparisons incorporated intra-animal baseline and postdrug datasets. For ANOVAs, post hoc (Holm-Sidak) pairwise multiple comparisons were performed to identify which groups were significantly different from one another. Significance was established using an $\alpha$ criterion of $p=0.05$. In the figures, ${ }^{\star} p<$ $0.05,{ }^{* *} p<0.01$, and ${ }^{* * *} p<0.001$, respectively. In the text, reported sample sizes denote the number of fish for each experimental group, which is also indicated by the numbers at the base of the bars in bar graphs within the figures.

\section{Results}

Behavioral and fictive correspondence in developmental switch of locomotor pattern

The developmental switch in the locomotor pattern from long to short swimming episodes has been demonstrated via peripheral nerve recordings in paralyzed larvae (Thirumalai and Cline, 2008), which is termed a fictive preparation. However, this switch has been characterized (Buss and Drapeau, 2001) but not quantified in free-swimming larvae. To this aim, we adapted opensource software, Ctrax (Branson et al., 2009), to individually track multiple zebrafish larvae in a group setting (Fig. $1 A$ ). This high-throughput tracking algorithm allows for the assessment of a suite of behavioral classifications that include an array of locomotor parameters (Branson et al., 2009). We focused on two behavioral locomotor parameters for each individual larva: (1) total distance traveled (centimeters in $10 \mathrm{~min}$ ), obtained from the sum of instantaneous speeds during swimming episodes divided by the frame rate (Fig. $1 B$ ), to assess the amount of freeswimming activity (Fig. $1 B$ ); and (2) mean swimming episode duration (milliseconds), obtained as the mean duration from the onset to offset of each swimming episode (Fig. $1 C$, expansion of $5 \mathrm{~s}$ from ${ }^{*}$ in $B$ ) across all identified swimming episodes (see Materials and Methods) in the speed function (Fig. $1 B$ ), to assess the locomotor pattern during free-swimming activity (Fig. 1C). The goal of these behavioral assessments was to compare the developmental switch in the free-swimming episodic locomotor pattern (Buss and Drapeau, 2001) to that of neural activity during fictive swimming.

Free-swimming larvae at $3 \mathrm{dpf}$ traveled significantly less distance $(4.7 \pm 11 \mathrm{~cm}, n=210)$ than did $4 \mathrm{dpf}$ larvae $(38 \pm 42 \mathrm{~cm}$, $\left.n=170 ; t_{(378)}=-11.2, p<0.001\right)$. In terms of the locomotor pattern, we found a correspondence between behavioral (Beh) (Fig. 1C) and fictive (Fic) (Fig. 1D) episode durations in the developmental switch from long to short swimming episodes between 3 dpf (Beh, $1014 \pm 684$ ms, $n=56$; Fic, $987 \pm 364$ ms, $n=$
10) and $4 \mathrm{dpf}$ (Beh, $248 \pm 59 \mathrm{~ms}, n=120$; Fic, $241 \pm 57 \mathrm{~ms}, n=$ 11). A two-way ANOVA revealed a significant effect of age $\left(F_{(1,193)}=74, p<0.001\right)$ but not preparation type (Beh vs Fic, $F_{(1,193)}=0.03, p=0.85$ ) (Fig. $1 E$ ). This behavioral and fictive correspondence suggests that the difference in the episodic locomotor pattern between 3 and $4 \mathrm{dpf}$ is attributable to an intrinsic development of the nervous system rather than a byproduct of extrinsic behavioral factors, which are not present in the fictive preparation.

The developmental switch from long to short swimming episodes did not begin over the time course of our recording sessions (9:00 A.M. to 6:00 P.M.) at $3 \mathrm{dpf}$, because the immature behavioral phenotype of long episode durations remained unchanged $\left(t_{(21)}=0.78, p=0.45\right)$ from the morning (9:00 A.M. to 11:00 A.M., $1228 \pm 893 \mathrm{~ms}, n=11$ ) to early evening (4:00 P.M. to 6:00 P.M., $1024 \pm 315 \mathrm{~ms}, n=12$ ). Strikingly, the switch occurred by the morning at $4 \mathrm{dpf}$, because the short episode durations at this time were not significantly different from those at the end of the recording day at $4 \mathrm{dpf}$ (9:00 A.M. to 11:00 A.M., $270 \pm 59 \mathrm{~ms}, n=$ 24; 4:00 P.M. to 6:00 P.M., $255 \pm 55 \mathrm{~ms}, n=30 ; t_{(52)}=0.97, p=$ $0.34)$. These data demonstrate that the developmental switch in the episodic locomotor pattern is rapid, occurring at some time window between the late evening at $3 \mathrm{dpf}$ and the early morning at $4 \mathrm{dpf}$.

\section{Blocking endogenous $D_{4} R$ signaling prevents maturation of the locomotor pattern}

To initially investigate whether the DDT is involved in conferring the mature episodic locomotor pattern, we tested the hypothesis that endogenous DAergic signaling is required to initiate the developmental switch to short swimming episodes. We systemically incubated larvae in specific DAR antagonists $\left(\mathrm{D}_{1} \mathrm{R}, \mathrm{SCH}-23390\right.$; $\mathrm{D}_{2} / \mathrm{D}_{3} \mathrm{R}$, raclopride; $\left.\mathrm{D}_{4} \mathrm{R}, \mathrm{L}-745,870\right)$ before and during the natural timing of the switch (3-4 dpf) and assessed resultant behavioral and fictive locomotor patterns at $4 \mathrm{dpf}$ (Fig. $2 \mathrm{~A}$ ). All DAR antagonists were administered at $10 \mu \mathrm{M}$, informed by initial concentration-response experiments (data not shown), in the presence of $0.1 \%$ DMSO to facilitate drug delivery (Rihel et al., 2010).

The DAR antagonists differentially affected the total distance traveled by $4 \mathrm{dpf}$ larvae (one-way ANOVA; $F_{(3,256)}=6.85, p<$ $0.001)$ (Fig. $2 B 1, B 2)$. Compared with DMSO vehicles $(38 \pm 41$ $\mathrm{cm}, n=60)$, there was no change in the total distance traveled by blocking $\mathrm{D}_{2} / \mathrm{D}_{3} \operatorname{Rs}(37 \pm 31 \mathrm{~cm}, n=70 ; p=0.79)$ (Fig. 2B1,B2). Conversely, blocking either $\mathrm{D}_{1}$ Rs or $\mathrm{D}_{4}$ Rs reduced the total distanced traveled $\left(\mathrm{D}_{1} \mathrm{R}, 24 \pm 30 \mathrm{~cm}, n=60, p<0.05 ; \mathrm{D}_{4} \mathrm{R}, 17 \pm 26\right.$ $\mathrm{cm}, n=70, p<0.001$ ) (Fig. 2B1,B2); note that both groups traveled significantly more than untreated 3 dpf larvae $\left(D_{1} R, p<\right.$ $\left.0.001 ; \mathrm{D}_{4} \mathrm{R}, p<0.001\right)$. This demonstrates that endogenous $\mathrm{D}_{1} \mathrm{R}$ and $\mathrm{D}_{4} \mathrm{R}$ signaling contribute to increased locomotion at $4 \mathrm{dpf}$ but that other contributions, such as from serotonergic signaling (Brustein et al., 2003), likely contribute to the differences in the amount of free-swimming of 3 and $4 \mathrm{dpf}$ larvae.

A two-way ANOVA revealed a significant effect on episode durations of $4 \mathrm{dpf}$ larvae for DAR antagonist incubations $\left(F_{(3,216)}=\right.$ $40, p<0.001$ ) but not preparation type (Beh vs Fic, $F_{(3,216)}=$ $1.30, p=0.26$ ) (Fig. 2C1,C2). The episode durations produced by larvae incubated in $\mathrm{D}_{1} \mathrm{R}$ antagonists (Beh, $470 \pm 249 \mathrm{~ms}, n=43$; Fic, $348 \pm 134 \mathrm{~ms}, n=8$ ) or $\mathrm{D}_{2} / \mathrm{D}_{3} \mathrm{R}$ antagonists (Beh, $298 \pm 104$ $\mathrm{ms}, n=62$; Fic, $268 \pm 108 \mathrm{~ms}, n=7)$ were not significantly different from DMSO vehicles (Beh, $253 \pm 52 \mathrm{~ms}, n=44$; Fic, $264 \pm 67 \mathrm{~ms}, n=7 ; p=0.11$ and $p=0.79$, respectively) (Fig. $2 C 1, C 2)$. In contrast, larvae incubated in $\mathrm{D}_{4} \mathrm{R}$ antagonists produced significantly longer episode durations (Beh, $1199 \pm 657$ 


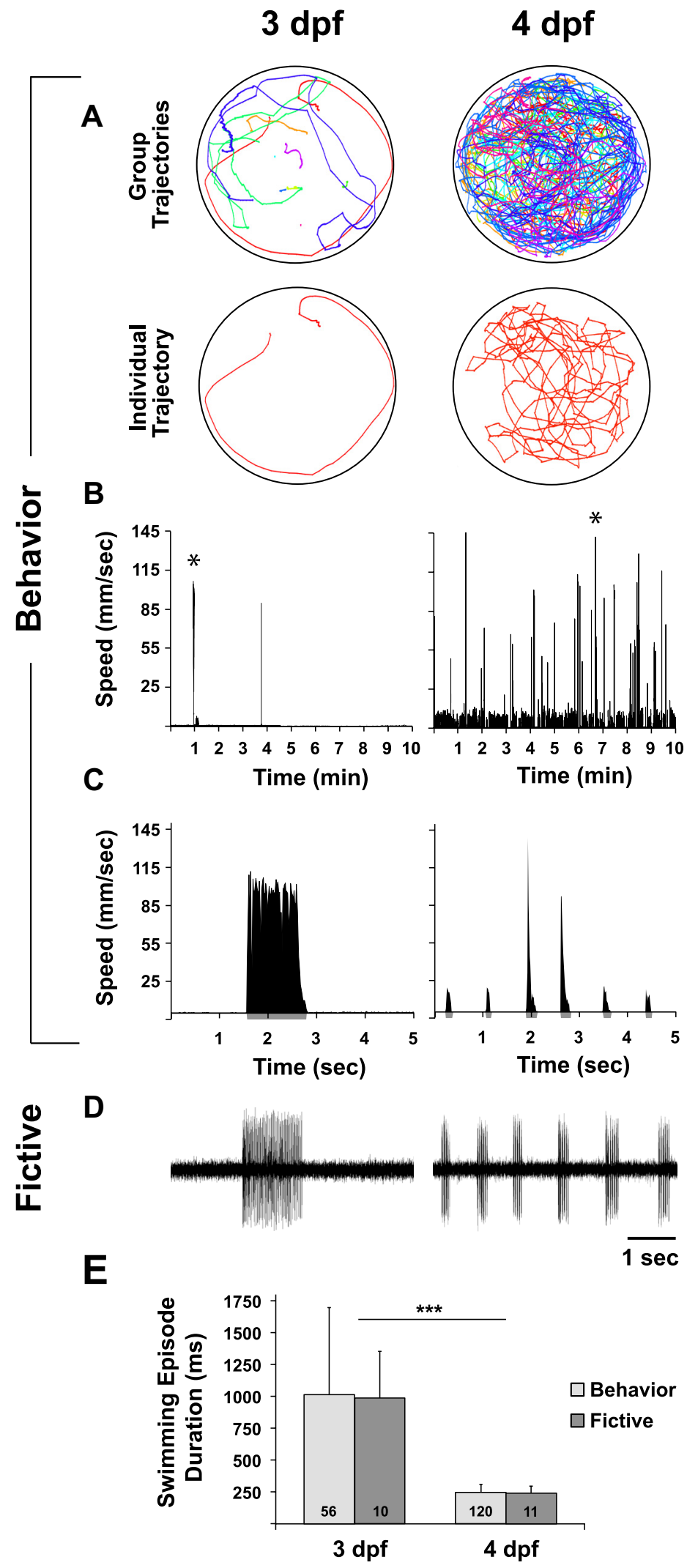

Figure 1. Behavioral and fictive correspondence in developmental switch of locomotor pattern. $A-E$, Comparison of behavioral $(\boldsymbol{A}-\boldsymbol{C})$ and fictive (D) swimming patterns between $3 \mathrm{dpf}$ (left column) and $4 \mathrm{dpf}$ (right column). $\boldsymbol{A}$, Free-swimming trajectory plots. Top row, Group trajectories from 10 larvae in the same $50 \mathrm{~mm}$ arena. Bottom row, Unique trajectory of an individual larva from the group trajectory above. $\boldsymbol{B}$, Speed function for an individual larva over the course of the $10 \mathrm{~min}$ record, from which total distance traveled and swimming episode durations were calculated. C, Expansion of $5 \mathrm{~s}$ from * in $\boldsymbol{B}$ to show the locomotor pattern of discrete swimming episodes. Gray regions below $x$-axis represent episode durations detected from filter settings (see Materials and Methods). $\boldsymbol{D}$, Voltage traces of fictive swimming from extracellular peripheral nerve ms, $n=52$; Fic, $1043 \pm 294 \mathrm{~ms}, n=8)$ than DMSO vehicles $(p<0.001)$ (Fig. $2 C 1, C 2)$. Moreover, the $\mathrm{D}_{4} \mathrm{R}$ antagonist prevented the maturation of the locomotor pattern, because the induced long episode durations were indistinguishable $(p=0.47)$ from that of $3 \mathrm{dpf}$ larvae (Fig. $2 C 2)$. These results suggest that endogenous $\mathrm{D}_{4} \mathrm{R}$ signaling mediates the advent of short swimming episodes.

\section{Blocking endogenous $D_{4} R$ signaling} reverts the mature locomotor pattern to an immature pattern

We next examined whether endogenous $\mathrm{D}_{4} \mathrm{R}$ signaling is required not only to initiate but also to maintain the mature locomotor pattern. To test this hypothesis, we systemically incubated larvae in specific DAR antagonists subsequent to the developmental switch (4-5 dpf) (Fig. 3A), such that larvae were already exhibiting increased locomotion in the form of short and directed swimming episodes. Resultant behavioral and fictive locomotor patterns were assessed at $5 \mathrm{dpf}$ (Fig. $3 A$ ).

The DAR antagonists differentially affected the total distance traveled by $5 \mathrm{dpf}$ larvae (one-way ANOVA, $F_{(3,246)}=20$; $p<0.001$ ) (Fig. 3B1,B2). Compared with DMSO vehicles $(36 \pm 18 \mathrm{~cm}, n=60)$, blocking $\mathrm{D}_{2} / \mathrm{D}_{3}$ Rs after the switch increased the total distanced traveled (54 \pm $27 \mathrm{~cm}, n=60 ; p<0.001$ ) (Fig. 3B1,B2), which was not observed when administered before the switch (Fig. 2B1,B2). Conversely, blocking either $\mathrm{D}_{1}$ Rs or $\mathrm{D}_{4} \mathrm{Rs}$ reduced the total distanced traveled $\left(D_{1} R\right.$, $26 \pm 27 \mathrm{~cm}, n=60, p<0.05 ; \mathrm{D}_{4} \mathrm{R}, 24 \pm$ $23 \mathrm{~cm}, n=70, p<0.01$ ) (Fig. 3B1,B2), similar to when the antagonists were given before the switch (Fig. 2B1,B2). These data suggest that, at $5 \mathrm{dpf}$, endogenous $\mathrm{D}_{1} \mathrm{R}$ and $\mathrm{D}_{4} \mathrm{R}$ signaling contribute to increased locomotion, whereas endogenous $\mathrm{D}_{2} / \mathrm{D}_{3} \mathrm{R}$ signaling suppresses the initiation of locomotion.

A two-way ANOVA revealed an effect on episode durations of $5 \mathrm{dpf}$ larvae for DAR antagonist incubations $\left(F_{(3,246)}=\right.$ $31, p<0.001)$ but not preparation type (Beh vs Fic, $F_{(3,246)}=0.65, p=0.42$ ) (Fig. $3 C 1, C 2)$. The episode durations produced by larvae incubated in $\mathrm{D}_{1} \mathrm{R}$ antagonists (Beh, $357 \pm 286 \mathrm{~ms}, n=46$; Fic, $383 \pm$

\section{$\longleftarrow$}

recordings. $\boldsymbol{E}$, Mean behavioral (light gray bars) and fictive (dark gray bars) swimming episode durations (milliseconds) at 3 and $4 \mathrm{dpf}$. Numbers at the base of bars in bar graph denote the sample sizes of fish for each experimental group. ${ }^{* *} p<$ 0.001 . 

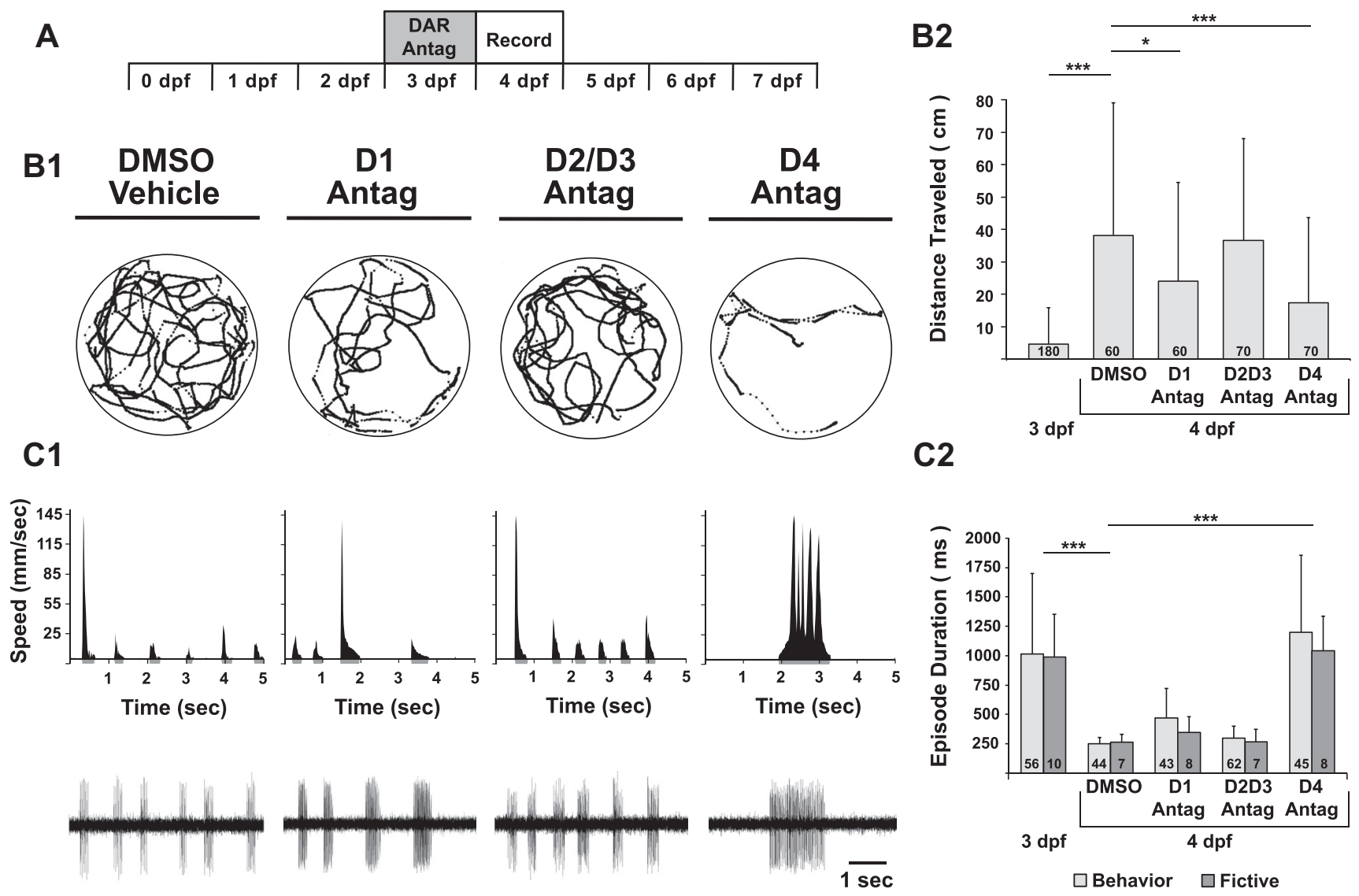

Figure 2. Blocking endogenous $D_{4} R$ signaling prevents maturation of the locomotor pattern. $A$, Timeline of experimental paradigm, in which larvae are incubated in DAR antagonists at $3 \mathrm{dpf}$, and behavioral and fictive locomotor patterns are assessed at $4 \mathrm{dpf}$. B1, Representative trajectory plot of an individual larva (from a group of 10 larvae in the same arena) over 10 min, depicting total activity per individual for each experimental group (DMSO, $D_{1}$ antagonist, $D_{2} / D_{3}$ antagonist, $D_{4}$ antagonist). Labels for experimental groups also correspond to data in $C 1$. Circles surrounding trajectories denote the periphery of the 50-mm-diameter arena. B2, Mean total distance traveled per individual (centimeters). C1, Behavioral (top row) and fictive (bottom row) locomotor pattern of discrete swimming episodes. Top row, Speed function from individual in B1. Gray regions below $x$-axis represent resultant episode durations detected from filter settings (see Materials and Methods). Bottom row, Voltage traces of fictive swimming from extracellular peripheral nerve recordings. (2, Mean behavioral (lights gray bars) and fictive (dark gray bars) swimming episode durations (milliseconds) at $3 \mathrm{dpf}$ and after pharmacological treatments at $4 \mathrm{dpf}$. Numbers at the base of bars in bar graphs denote the sample sizes of fish for each experimental group. ${ }^{*} p<0.05$; ${ }^{* * *} p<0.001$.

$256 \mathrm{~ms}, n=7$ ) or $\mathrm{D}_{2} / \mathrm{D}_{3} \mathrm{R}$ antagonists (Beh, $253 \pm 114 \mathrm{~ms}, n=$ 59; Fic, $261 \pm 57 \mathrm{~ms}, n=7$ ) were not significantly different from DMSO vehicles (Beh, $234 \pm 60 \mathrm{~ms}, n=59$; Fic, $245 \pm 53 \mathrm{~ms}, n=$ $6 ; p=0.14$ and $p=0.84$, respectively) (Fig. 3C1,C2). Conversely, larvae incubated in $\mathrm{D}_{4} \mathrm{R}$ antagonists produced significantly longer episode durations (Beh, $871 \pm 492 \mathrm{~ms}, n=64$; Fic, $1022 \pm$ $493 \mathrm{~ms}, n=7)$ than DMSO vehicles $(p<0.001)$ (Fig. 3C1,C2). Moreover, the $\mathrm{D}_{4} \mathrm{R}$ antagonist reversed the developmental switch in the locomotor pattern, relegating $5 \mathrm{dpf}$ larvae to long episode durations that were not significantly different from that of $3 \mathrm{dpf}$ larvae $(p=0.72)$ (Fig. 3C2). These results demonstrate that $\mathrm{D}_{4} \mathrm{R}$ signaling is necessary to maintain the mature locomotor pattern of short swimming episodes at $5 \mathrm{dpf}$.

$D_{4} R$ signaling in the spinal cord is sufficient to induce the mature locomotor pattern of short swimming episodes

Having elucidated that systemic block of $\mathrm{D}_{4} \mathrm{R}$ signaling can both prevent (Fig. 2) and reverse (Fig. 3) the developmental switch in the locomotor pattern, we next investigated whether the anatomical site of action is in the spinal cord. To test this, we compared the fictive locomotor pattern of intact animals at 4-7 dpf compared with those that were spinalized, in which the spinal cord was isolated from the brain to remove all descending inputs, including the DDT. Because fictive swimming in spinalized prep- arations does not occur spontaneously but can be elicited with NMDA (McDearmid and Drapeau, 2006), we added NMDA (50 $\mu \mathrm{M})$ to induce fictive locomotor output and subsequently tested the effects of exogenous application of DAergic agonists. To more directly compare intact and spinalized fictive swimming, we assessed NMDA-induced, in addition to spontaneous, fictive swimming in intact animals.

There was a significant difference in episode durations among the experimental groups $\left(F_{(3,65)}=23, p<0.001\right)$. In intact preparations, episode durations were not significantly different $(p=$ $0.61)$ between spontaneous $(241 \pm 57 \mathrm{~ms}, n=11)$ and NMDAinduced ( $300 \pm 186 \mathrm{~ms}, n=21$ ) fictive swimming (Fig. 4A,B). Conversely, significantly longer episode durations were produced by spinalized preparations $(1871 \pm 1285 \mathrm{~ms}, n=30$; $p<0.001$ ) (Fig. $4 A, B$ ), reminiscent of the immature locomotor pattern of intact $3 \mathrm{dpf}$ larvae (Fig. $1 D, E$ ). This demonstrates that some descending input from the brain is required to confer short NMDA-induced swimming episodes. Next, we tested whether supplying exogenous DA to the putatively DA-deprived transected spinal cord could sculpt NMDA-induced locomotor activity to better resemble that of the intact animal. Application of exogenous DA $(1 \mu \mathrm{M})$ to spinalized preparations significantly shortened NMDA-induced episode durations $(601 \pm 251 \mathrm{~ms}$, $n=7)$ when compared with NMDA application alone $(p<$ 
A

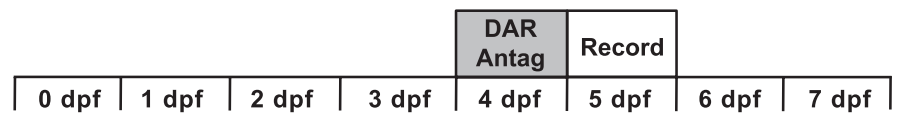

B1

DMSO Vehicle

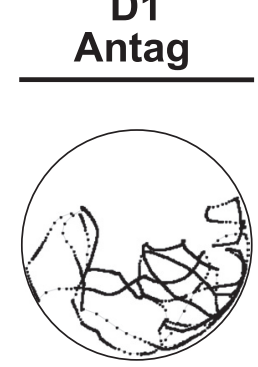

C1
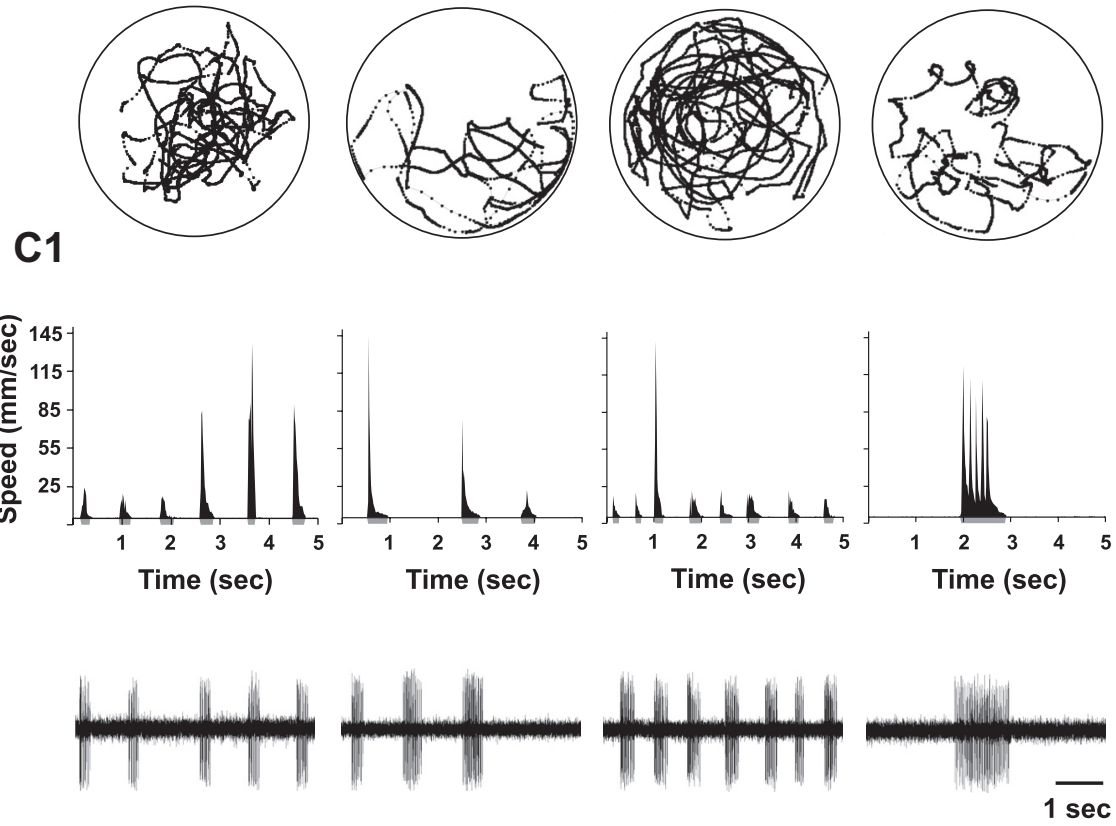

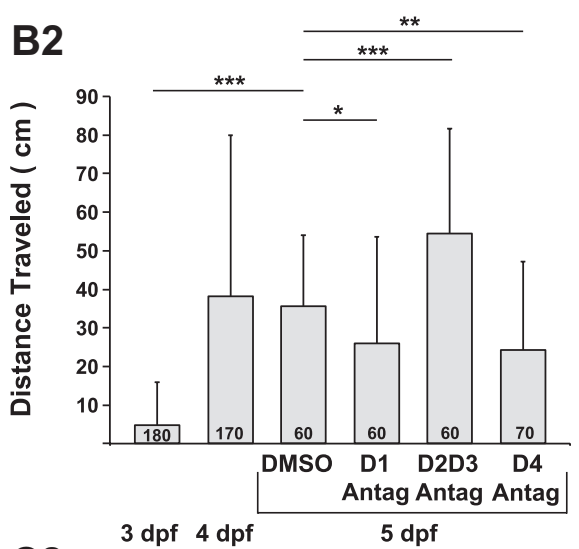

\section{C2}

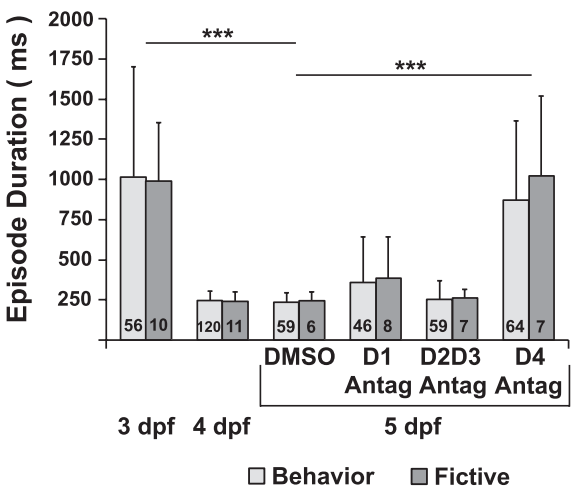

Figure 3. Blocking endogenous $D_{4} R$ signaling reverts the mature locomotor pattern to an immature pattern. $A$, Timeline of experimental paradigm, in which larvae are incubated in DAR antagonists at $4 \mathrm{dpf}$, and resultant behavioral and fictive locomotor patterns are assessed at $5 \mathrm{dpf}$. B1, Representative trajectory plot of an individual larva (from a group of 10 larvae in the same arena) over $10 \mathrm{~min}$, depicting total activity per individual for each experimental group ( $\mathrm{DMSO}, \mathrm{D}_{1}$ antagonist, $\mathrm{D}_{2} / \mathrm{D}_{3}$ antagonist, $\mathrm{D}_{4}$ antagonist). Labels for experimental groups also correspond to data in C1. Circles surrounding trajectories denote the periphery of the 50-mm-diameter arena. B2, Mean total distance traveled per individual (centimeters). C1, Behavioral (top row) and fictive (bottom row) locomotor pattern of discrete swimming episodes. Top row, Speed function from individual in $\boldsymbol{B}$. Gray regions below $x$-axis represent resultant episode durations detected from filter settings (see Materials and Methods). Bottom row, Voltage traces of fictive swimming from extracellular peripheral nerve recordings. C2, Mean behavioral (lights gray bars) and fictive (dark gray bars) swimming episode durations (milliseconds) at $3 \mathrm{dpf}, 4 \mathrm{dpf}$, and after pharmacological treatments at $5 \mathrm{dpf}$. Numbers at the base of bars in bar graphs denote the sample sizes of fish for each experimental group. ${ }^{*} p<0.05 ;{ }^{* *} p<0.01 ;{ }^{* * *} p<0.001$.

A

\section{Intact Spontaneous}
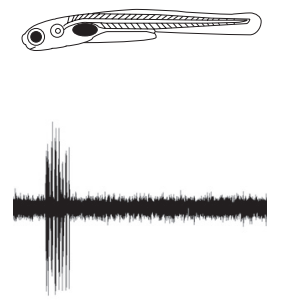

Intact NMDA

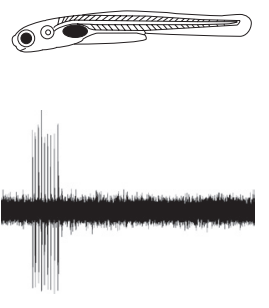

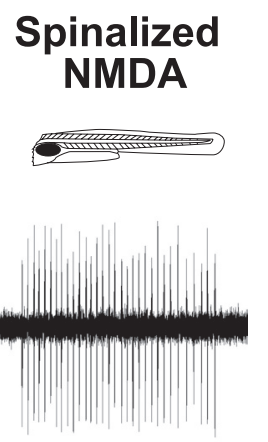
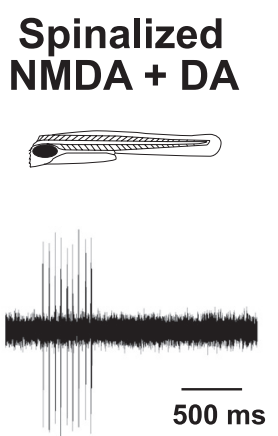

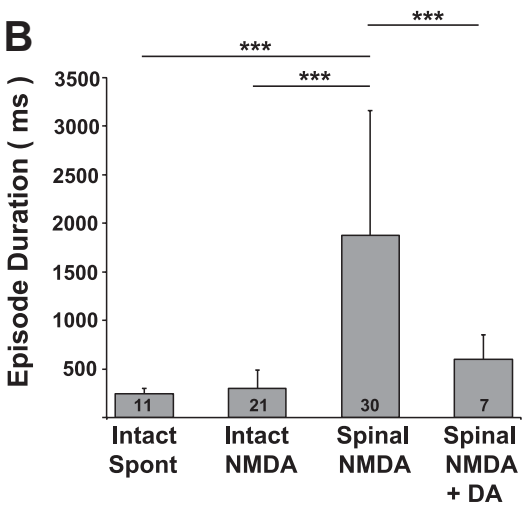

Figure 4. DAergic signaling in the spinal cord is sufficient to induce the mature locomotor pattern of short swimming episodes. $A$, Voltage traces of fictive swimming from extracellular peripheral nerve recordings from intact and spinalized preparations at $4-7 \mathrm{dpf}$. $\boldsymbol{B}$, Mean fictive swimming episode durations (milliseconds). Numbers at the base of bars in bar graph denote the sample sizes of fish for each experimental group. ${ }^{* *} p<0.001$.

0.001) (Fig. 4A,B). This result demonstrates that DA can act directly in the spinal cord to shorten fictive swimming episodes.

To determine whether DA acts via excitatory $\left(D_{1}\right.$-like: $D_{1} R$, $\left.D_{5} R\right)$ or inhibitory $\left(D_{2}\right.$-like: $\left.D_{2} R, D_{3} R, D_{4} R\right)$ DARs in the spinal cord to shorten NMDA-induced fictive episode durations, DARspecific agonists $\left(\mathrm{D}_{1}\right.$-like, SKF-38393; $\mathrm{D}_{2}$-like, quinpirole; or $\mathrm{D}_{4} \mathrm{R}, \mathrm{PD} 168077$ ) were applied to spinalized preparations of 4-7 dpf larvae. There was a significant difference among the pharmacological treatments $\left(F_{(4,55)}=8.4, p<0.001\right)$. Compared with 
A

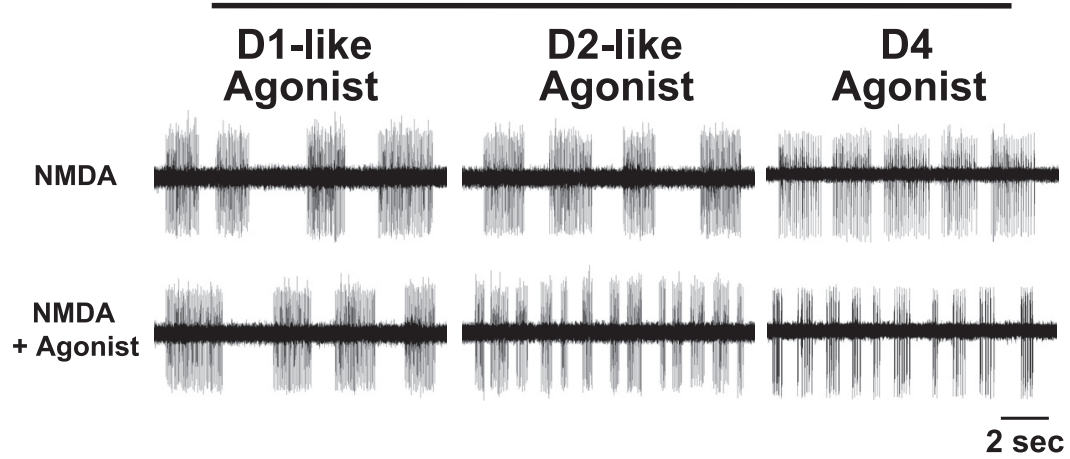

B

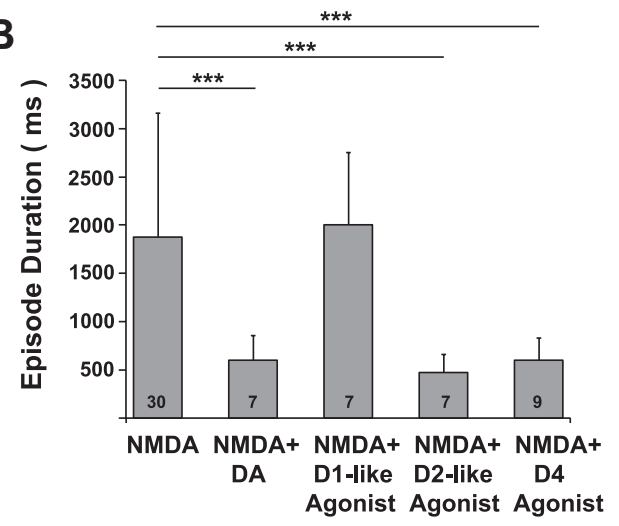

Figure 5. $D_{4} R$ signaling in the spinal cord is sufficient to induce the mature locomotor pattern of short swimming episodes. $A$, Voltage traces of fictive swimming from extracellular peripheral nerve recordings from spinalized preparations at $4-7 \mathrm{dpf}$ in NMDA alone (top traces) and NMDA + agonist (bottom traces). $\boldsymbol{B}$, Mean fictive swimming episode durations (milliseconds). Numbers at the base of bars in bar graph denote the sample sizes of fish for each experimental group. ${ }^{* * *} p<0.001$.

NMDA alone, the $\mathrm{D}_{1}$-like agonist $(1 \mu \mathrm{M})$ had no effect on episode duration ( $1999 \pm 750 \mathrm{~ms}, n=7 ; p=0.34$ ), whereas the $\mathrm{D}_{2}$-like agonist $(1 \mu \mathrm{M})$ significantly shortened episode durations (475 \pm $183 \mathrm{~ms}, n=7 ; p<0.001$ ) (Fig. $5 A, B$ ), similar to exogenous DA (Fig. $5 B$ ). Moreover, application of a specific $\mathrm{D}_{4} \mathrm{R}$ agonist $(1 \mu \mathrm{M})$ was sufficient to significantly shorten episode durations (598 \pm $234 \mathrm{~ms}, n=9$; $p<0.001$ ) (Fig. 5A,B), similar to that of exogenous DA and the broad-spectrum $\mathrm{D}_{2}$-like agonist (Fig. $5 B$ ). This result demonstrates that $\mathrm{D}_{4} \mathrm{R}$ signaling in the spinal cord is sufficient to shorten fictive episodes.

\section{Selective chemogenetic ablation of otpb neurons}

The evidence that DAergic signaling in the spinal cord is sufficient to shorten fictive swimming episodes (Figs. 4, 5) suggests a critical role for otp neurons in sculpting the mature episodic locomotor pattern, because otp neurons provide the exclusive source of spinal DA (Kastenhuber et al., 2010; Tay et al., 2011). To explore this possibility, we used a transgenic line that drives expression in DAergic otp $b$ neurons in the caudal ventral diencephalon, which coexpresses TH and dat and forms part of the DDT (Fujimoto et al., 2011). In addition, this transgenic line drives expression in a non-DAergic neurosecretory otp $b$ population in the rostral diencephalon (Fujimoto et al., 2011). To test the hypothesis that $o t p b$ cells contribute to conferring the mature episodic locomotor pattern, we performed selective, cell-autonomous chemogenetic ablation of $o t p b$ cells in $\operatorname{Tg}(\text { otpb.A:nfsB-egfp })^{\mathrm{zc} 77}$ larvae via the Ntr:Mtz system (Curado et al., 2008; Pisharath and Parsons, 2009).

Application of Mtz to the aqueous environment from 1 to 4 dpf (Fig. 6A) induced selective cell death of $o t p b$ neurons in $\operatorname{Tg}(\text { otpb.A:nfsB-egfp })^{\mathrm{zc} 77}$ larvae (Fig. 6B,C). A marked increase of TUNEL staining in Mtz-treated $\mathrm{Ntr}^{+}$larvae $\left(\mathrm{Ntr}^{+} / \mathrm{Mtz}^{+}\right)$was observed when compared with untreated $\mathrm{Ntr}^{+}$siblings $\left(\mathrm{Ntr}^{+} /\right.$ $\mathrm{Mtz}^{-}$) or Mtz-treated WT larvae (WT/Mtz ${ }^{+}$) (Fig. 6B). Moreover, the increased TUNEL staining in $\mathrm{Ntr}^{+} / \mathrm{Mtz}^{+}$larvae colocalized to both rostral non-DAergic and caudal DAergic diencephalic $\mathrm{GFP}^{+}$populations (Fig. $6 \mathrm{C}$ ). By $3 \mathrm{dpf}, \mathrm{WT} / \mathrm{Mtz}^{+}$and $\mathrm{Ntr}^{+} / \mathrm{Mtz}^{-}$larvae showed robust dat mRNA expression in the ventral diencephalon (Fig. 6D), which colocalizes with the DAergic otp $b$ population (Fujimoto et al., 2011). Conversely, there was a marked absence of dat mRNA expression in the ventral diencephalon of $\mathrm{Ntr}^{+} / \mathrm{Mtz}^{+}$larvae (Fig. 6D), coincident spatially with the $\mathrm{Ntr}^{+} / \mathrm{Mtz}^{+}$induced TUNEL staining (Fig. $6 \mathrm{~B}$, arrows in right). This indicates an $\mathrm{Ntr}^{+} / \mathrm{Mtz}^{+}$-mediated loss of putatively functional DAergic otp $b$ cells in the ventral diencephalon. Additionally, by $7 \mathrm{dpf}$, there was an absence of $\mathrm{GFP}^{+}$cells in $\mathrm{Ntr}^{+} /$ $\mathrm{Mtz}^{+}$larvae when compared with $\mathrm{Ntr}^{+} / \mathrm{Mtz}^{-}$siblings (Fig. $6 E$, left column), suggesting an Mtz-mediated loss of $\mathrm{Ntr}^{+}$cells. Many of the GFP ${ }^{+}$cells in the ventral diencephalon of $\mathrm{Ntr}^{+}$/ $\mathrm{Mtz}^{-}$siblings colocalize with $\mathrm{TH}^{+}$cells in the ventral diencephalon (Fig. 6E, top right), which suggests that Mtz treatment induced a loss of double-labeled $\mathrm{TH}^{+}$and $\mathrm{Ntr}^{+}\left(\mathrm{GFP}^{+}\right)$otpb DAergic cells (Fig. 6E). To determine the efficacy of $\mathrm{Ntr}^{+} / \mathrm{Mtz}^{+}$ mediated ablation of DAergic otp $b$ cells, we counted the number of cells in the diencephalon that expressed both TH and GFP, in $\mathrm{Ntr}^{+} / \mathrm{Mtz}^{-}$compared with $\mathrm{Ntr}^{+} / \mathrm{Mtz}^{+}$larvae. At $7 \mathrm{dpf}, \mathrm{Ntr}^{+} /$ $\mathrm{Mtz}^{-}$larvae had $26 \pm 3 \mathrm{TH}^{+} / \mathrm{GFP}^{+}$DAergic otp $b$ cells, which was significantly reduced to $3 \pm 0.9 \mathrm{TH}^{+} / \mathrm{GFP}^{+}$DAergic otpb cells in $\mathrm{Ntr}^{+} / \mathrm{Mtz}^{+}$larvae $(p<0.0001 ; n=11$ larvae per group; mean \pm SEM). Together, these results demonstrate that the Mtz regimen was effective in selectively ablating otp $b \mathrm{Ntr}^{+}$cells in $\operatorname{Tg}(\text { otpb.A: } n f s B-e g f p)^{\mathrm{zc} 77}$ larvae.

\section{Loss of $o t p b$ neurons prevents maturation of the locomotor pattern}

To test whether $o t p b$ neurons contribute to the developmental switch to the mature episodic locomotor pattern, we assessed the effect of ablating $o t p b$ neurons on the behavioral and fictive locomotor patterns at $5 \mathrm{dpf}$ (Fig. 7A). There was a significant difference in the total distance traveled among the experimental groups $\left(F_{(3,206)}=15, p<0.001\right)$. There were no significant differences among the total distance traveled by $\mathrm{Ntr}^{-} / \mathrm{Mtz}^{-}$(39 \pm $32 \mathrm{~cm}, n=50), \mathrm{Ntr}^{-} / \mathrm{Mtz}^{+}(40 \pm 32 \mathrm{~cm}, n=40)$ and $\mathrm{Ntr}^{+} /$ $\mathrm{Mtz}^{-}(42 \pm 25 \mathrm{~cm}, n=50)$ larvae $(p>0.76$ for all pairwise comparisons) (Fig. 7B1,B2). Conversely, selective chemogenetic ablation of $o t p b$ cells in $\mathrm{Ntr}^{+} / \mathrm{Mtz}^{+}$larvae significantly reduced the total distance traveled $(14 \pm 13 \mathrm{~cm}, n=60)$ compared with all other groups $(p<0.001)$ (Fig. 7B1,B2). In terms of the locomotor pattern, a two-way ANOVA revealed an effect on episode duration for treatment condition $\left(F_{(3,211)}=15, p<0.001\right)$ but not preparation type (Beh vs Fic, $F_{(3,211)}=1.49, p=0.22$ ). The stage-appropriate expression of short episodes was observed in both behavioral and fictive preparations from $\mathrm{Ntr}^{-} / \mathrm{Mtz}^{-}$(Beh, $315 \pm 82 \mathrm{~ms}, n=47$; Fic, $351 \pm 112 \mathrm{~ms}, n=7), \mathrm{Ntr}^{-} / \mathrm{Mtz}^{+}$ (Beh, $317 \pm 124 \mathrm{~ms}, n=37$; Fic, $379 \pm 116 \mathrm{~ms}, n=7$ ) and $\mathrm{Ntr}^{+} / \mathrm{Mtz}^{-}$(Beh, $298 \pm 212 \mathrm{~ms}, n=50$; Fic, $349 \pm 186 \mathrm{~ms}, n=$ 7) larvae ( $p>0.75$ for all pairwise comparisons) (Fig. 7C1,C2). Conversely, a significant increase in the durations of behavioral 


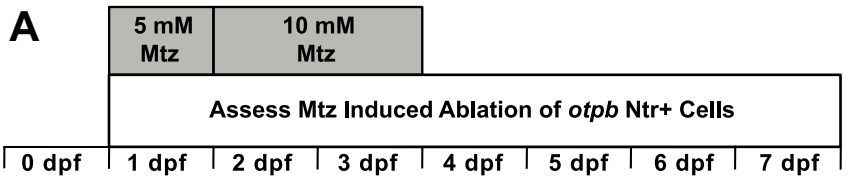

B

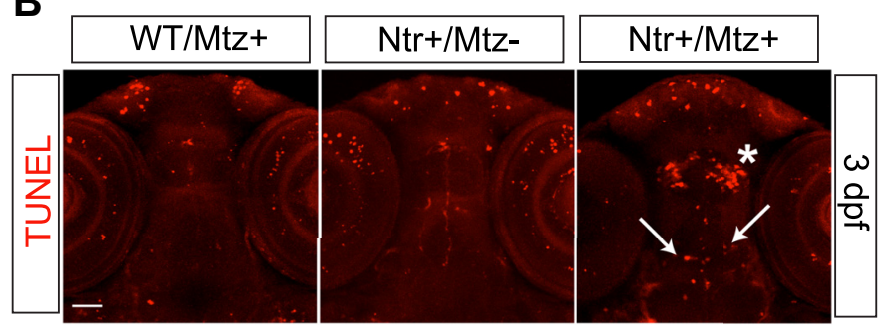

C

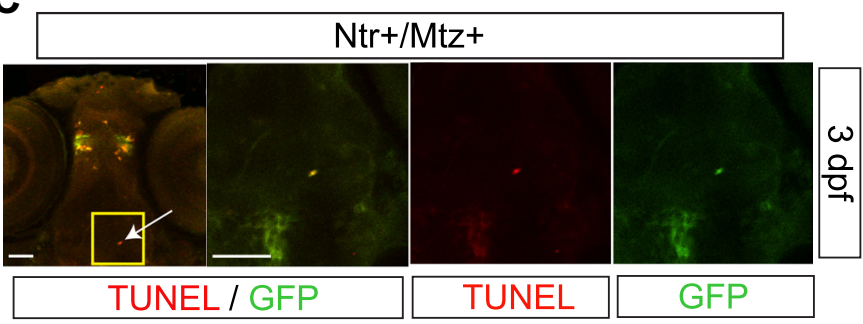

D

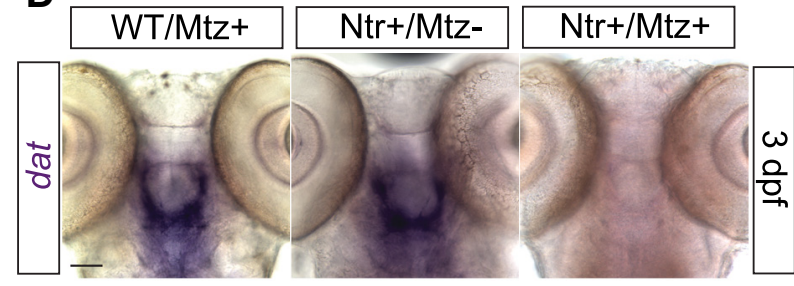

$\mathbf{E}$

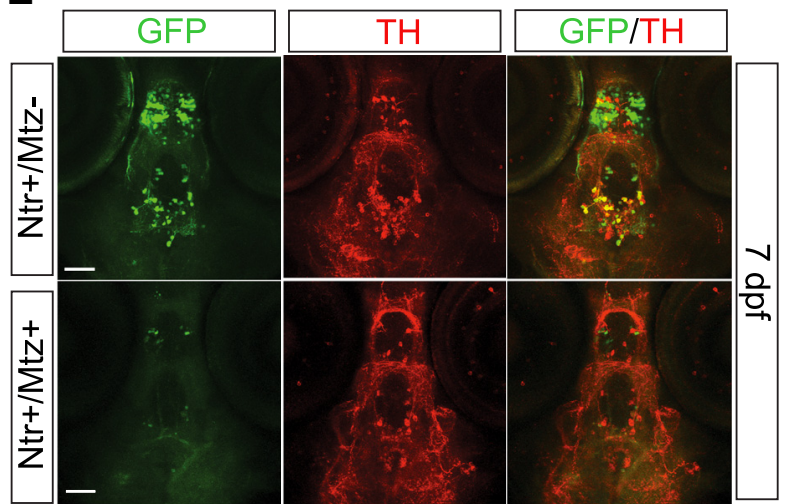

Figure 6. Selective chemogenetic ablation of otpb neurons. A, Timeline of experimental paradigm, in which larvae were incubated in $5 \mathrm{~mm}$ Mtz from 24 to $48 \mathrm{hpf}$ and $10 \mathrm{~mm} \mathrm{Mtz} \mathrm{from} 48$ to 96 hpf; assessment of cell death was investigated from 1 to $7 \mathrm{dpf}$. $\boldsymbol{B}-\boldsymbol{E}$, Ventral views in whole-mount $\mathrm{Tg}$ (otpb.A:nfsB-egfp) ${ }^{z c 77}$ larvae; rostral is to the top. Scale bars, $50 \mu \mathrm{m}$. $\boldsymbol{B}$, TUNEL signal in confocal z-stacks; asterisk denotes region of rostral non-DAergic otp ${ }^{+}$population, and arrows denote caudal DAergic otp $b^{+}$population. $\boldsymbol{C}$, Confocal images showing colocalization of TUNEL signal and GFP in larvae treated with Mtz. First panel shows z-stack image; yellow box indicates caudal ventral diencephalon, which is magnified in subsequent panels as single confocal slices; arrow denotes a GFP ${ }^{+}$and TUNEL stained cell. $\boldsymbol{D}$, Bright-field images of loss of s/c6a3 (dat) mRNA expression via in situ hybridization in whole-mount embryos. $\boldsymbol{E}$, Confocal $z$-stack images of double labeling for GFP and TH immunohistochemistry at $7 \mathrm{dpf}$.

and fictive episodes was observed in $\mathrm{Ntr}^{+} / \mathrm{Mtz}^{+}$larvae (Beh, $738 \pm 697 \mathrm{~ms}, n=56$; Fic, $897 \pm 311 \mathrm{~ms}, n=12)$ compared with all other groups $(p<0.001)$ (Fig. 7C1,C2). These results indicate that otpb cells contribute to the mature locomotor pattern of short episodes because their specific ablation perpetuates long episodes in $5 \mathrm{dpf}$ larvae.

\section{Removal of the caudal diencephalon reverts the mature} locomotor pattern to an immature pattern that is rescued by $\mathrm{D}_{4} \mathrm{R}$ agonism

Specific chemogenetic ablation of $o t p b$ cells included the rostral non-DAergic and caudal DAergic otp $b$ populations. To determine the relative contributions of DAergic and non-DAergic $o t p b^{+}$cells to the generation of short episodes, $\operatorname{Tg}(o t p b . A: n f s B-$ egfp ${ }^{\mathrm{zc} 77}$ larvae at 4-7 dpf were used to produce reduced preparations in which the nervous system was transected either just rostral to or just caudal to the DAergic $o t p b$ population. Middiencephalic-transected (MDT) (Fig. $8 \mathrm{~A}$ ) preparations lack nonDAergic otp $b$ cells and the ascending projections from DAergic $o t p b$ cells but retain DAergic otpb somata and their descending projections (i.e., including the DDT). Caudal-diencephalictransected (CDT) (Fig. $8 \mathrm{~A}$ ) preparations lack both DAergic and non-DAergic otp $b$ neurons but retain midbrain and hindbrain structures. The consequences of these transections were examined by measuring episode durations in fictive preparations.

Intact, MDT and CDT preparations exhibited spontaneous fictive swimming, but spinalized preparations did not (Fig. $8 \mathrm{~B}$ ). Spontaneous episode durations were significantly different among the intact, MDT, and CDT groups $\left(F_{(2,23)}=41, p<\right.$ $0.001)$. MDT preparations, which retain the DAergic otp $b$ population and the DDT, produced short spontaneous episode dura- tions $(320 \pm 187 \mathrm{~ms}, n=8)$ that were not significantly different from intact larvae $(333 \pm 211 \mathrm{~ms}, n=8 ; p=0.91)($ Fig. $8 B, D)$. CDT preparations, which lack DAergic otpb neurons, produced significantly longer spontaneous episode durations $(1105 \pm 231$ ms, $n=10$; $p<0.001$ ) (Fig. $8 B, D$ ) that were not significantly different $(p=0.40)$ from the episodes observed in $3 \mathrm{dpf}$ WT larvae (Fig. $1 D)$. These results demonstrate that neither the nonDAergic otpb population nor the ascending projections of the DAergic $o t p b$ population are necessary for the generation of short spontaneous episodes. Only the caudal diencephalic region, in which DAergic otpb neurons reside, is necessary for the production of short spontaneous episodes.

Next, we tested the hypothesis that $\mathrm{D}_{4} \mathrm{R}$ agonism would shorten the long episodes of CDT preparations to stageappropriate short episodes and that this would be recapitulated at the level of the spinal cord via spinalized preparations. Because spinalized preparations do not exhibit spontaneous fictive swimming (Fig. $8 \mathrm{~B}$ ), we compared NMDA-induced locomotor activity from intact, MDT, CDT, and spinalized preparations in $\operatorname{Tg}(\text { otpb.A:nfsB-egfp })^{z c 77}$ larvae (Fig. $8 C$ ). There was a significant difference in the NMDA-induced episode durations among these preparations $\left(F_{(5,36)}=9.18, p<0.001\right)$. The short NMDAinduced episode durations of intact $(317 \pm 250 \mathrm{~ms}, n=4)$ and MDT $(379 \pm 172 \mathrm{~ms}, n=7)$ preparations were not significantly different from one another $(p=0.82)$ or when compared with spontaneous episode durations of intact and MDT preparations ( $p=0.91$ and $p=0.53$, respectively) (Fig. $8 C, D$ ). Conversely, the duration of the NMDA-induced episodes generated by CDT $(1355 \pm 432 \mathrm{~ms}, n=12)$ and spinalized $(1310 \pm 830 \mathrm{~ms}, n=7)$ preparations were significantly longer $(p<0.001$ and $p<0.001$, respectively) when compared with intact or MDT preparations 
A
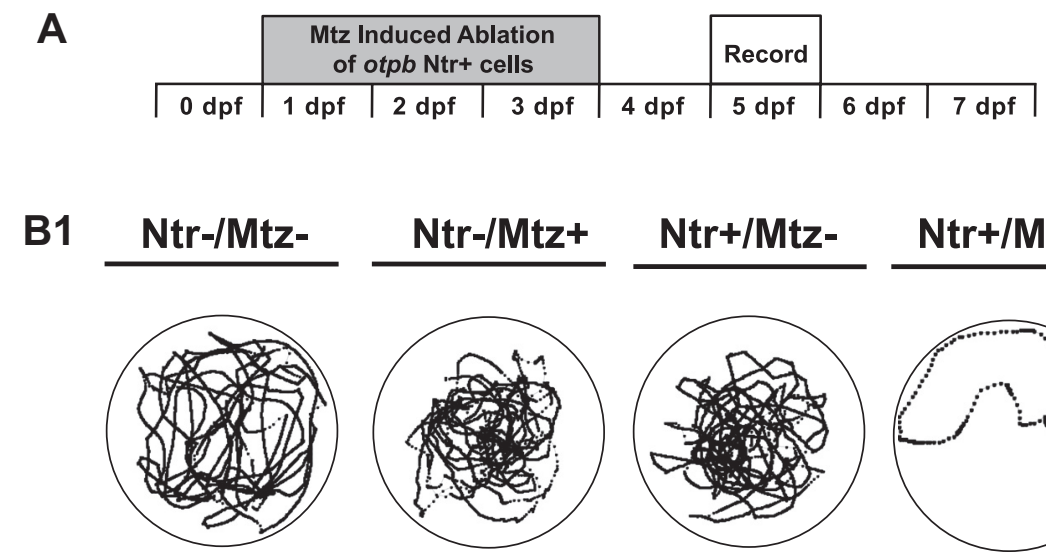

C1
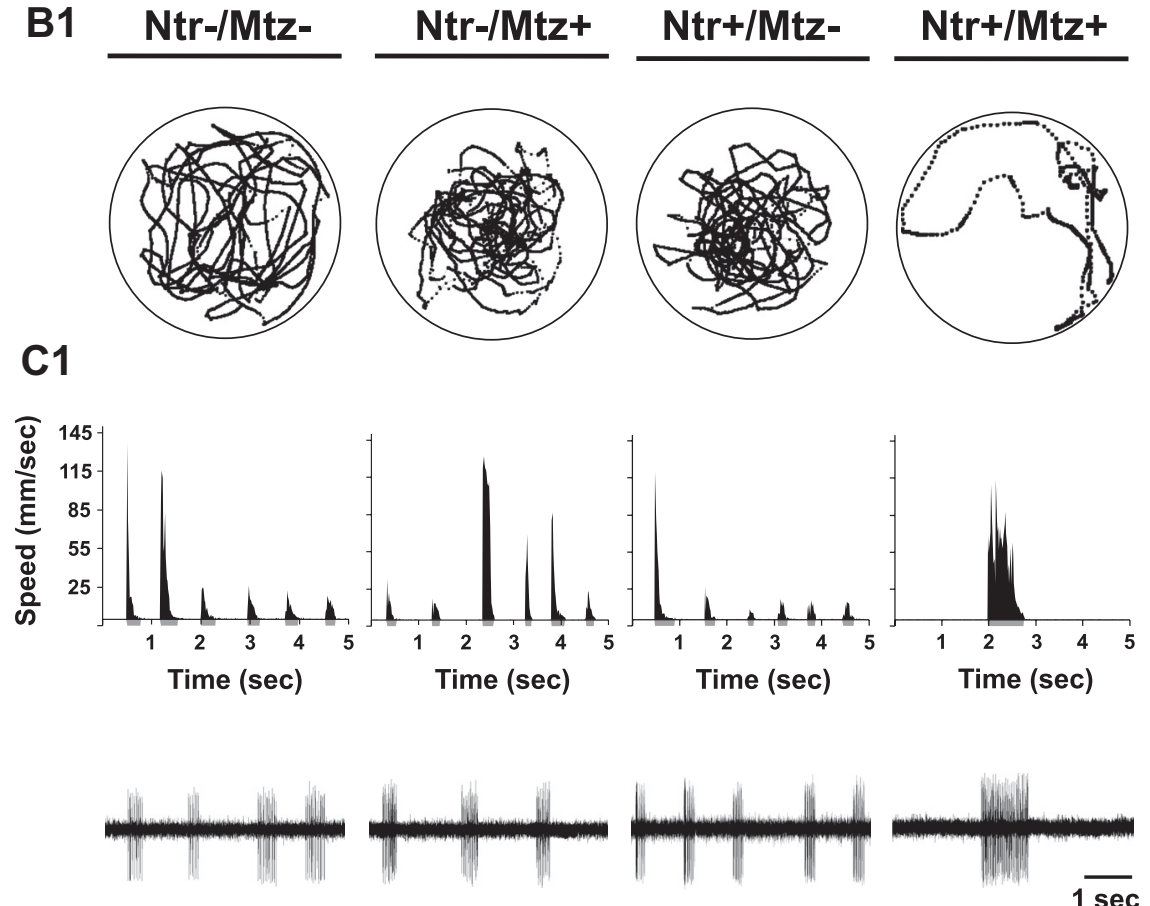
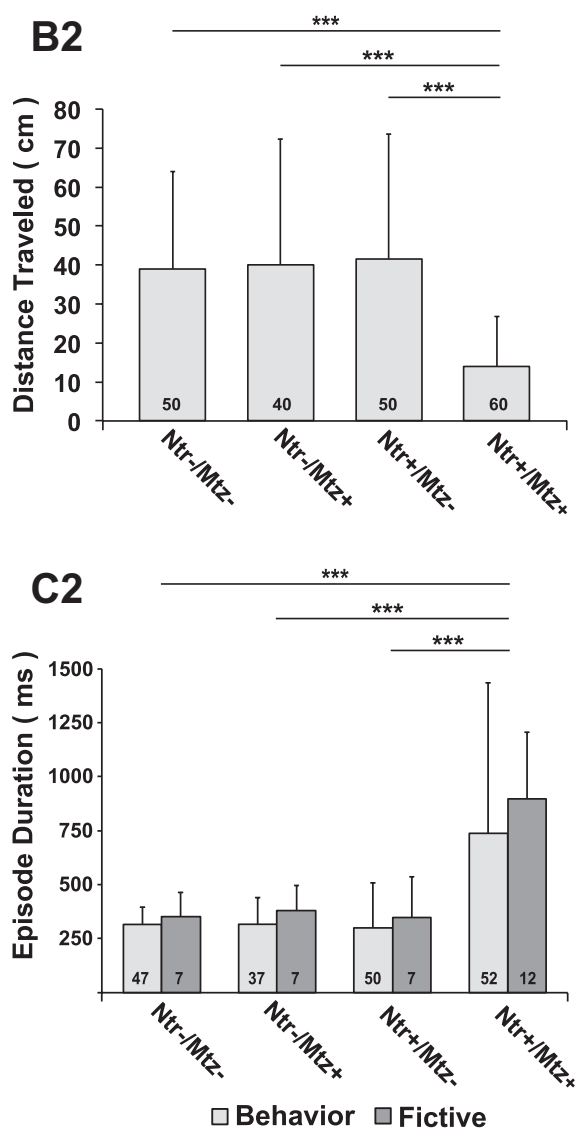

Figure 7. Loss of otpb neurons prevents maturation of the locomotor pattern. $A$, Timeline of experimental paradigm, in which otpb ${ }^{+}$cells are chemogenetically ablated via Mtz treatment from

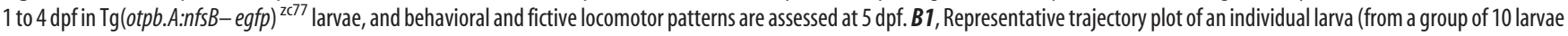
in the same arena) over $10 \mathrm{~min}$, depicting total activity per individual for each experimental group ( $\left.\mathrm{Ntr}{ }^{-} / \mathrm{Mtz}^{-}, \mathrm{Ntr}^{-} / \mathrm{Mtz}^{+}, \mathrm{Ntr}^{+} / \mathrm{Mtz}^{-}, \mathrm{Ntr}^{+} / \mathrm{Mtz}^{+}\right)$. Labels for experimental groups also correspond to data in C1. Circles surrounding trajectories denote the periphery of the 50-mm-diameter arena. B2, Mean total distance traveled per individual (centimeters). C1, Behavioral (top row) and fictive (bottom row) locomotor pattern of discrete swimming episodes. Top row, Speed function from individual in $\boldsymbol{B}$ 1. Gray regions below $x$-axis represent resultant episode durations detected from filter settings (see Materials and Methods). Bottom row, Voltage traces of fictive swimming from extracellular peripheral nerve recordings. (2, Mean behavioral (lights gray bars) and fictive (dark gray bars) swimming episode durations (milliseconds) at 5 dpf. Numbers at the base of bars in bar graphs denote the sample sizes of fish for each experimental group. ${ }^{* * *} p<0.001$.

(Fig. 8C,D). However, the long NMDA-induced episodes of CDT and spinalized preparations were not significantly different from one another $(p=0.85)$ or when compared with spontaneous episodes of CDT preparations ( $p=0.11$ and $p=0.47$, respectively) (Fig. $8 D$ ). Subsequent to NMDA-induced locomotor activation, the $\mathrm{D}_{4} \mathrm{R}$ agonist was exogenously applied and episode durations were measured in CDT and spinalized preparations (Fig. 8C,D). A change in the locomotor pattern from long to short episode durations was observed in both preparations $(\mathrm{CDT}$, $533 \pm 172 \mathrm{~ms}, n=6, p<0.001$; spinalized, $458 \pm 117 \mathrm{~ms}, n=5$, $p<0.001)$. This result demonstrates that, in the absence of the caudal diencephalon, $\mathrm{D}_{4} \mathrm{R}$ agonism is sufficient to produce short episodes and suggests that the DAergic otpb population is the critical caudal diencephalic component for conferring the mature episodic locomotor pattern.

\section{Discussion}

We explored the function of the evolutionarily conserved vertebrate DDT and found that it mediates the progression of locomotor development in zebrafish larvae. The developmental switch to the mature episodic locomotor pattern requires spinal $\mathrm{D}_{4} \mathrm{R}$ signaling that is driven by DAergic otpb cells comprising the DDT. Loss of DAergic input before the developmental switch led to a maintained immature locomotor pattern. Furthermore, continued DAergic input is necessary, because loss of $\mathrm{D}_{4} \mathrm{R}$ signaling after the developmental switch led to a reversion to the immature pattern. Our findings demonstrate a tonic requirement for central sources of DAergic signaling that act in the spinal cord, with implications for understanding the normal development of locomotion in vertebrates or effects from disease states.

\section{Behavioral and fictive correspondence in the locomotor pattern}

Although a myriad of behavioral studies in zebrafish larvae have assayed the amount of free-swimming activity under an array of paradigms (for review, see Tierney, 2011), only a few have assessed the actual locomotor pattern of swimming episodes during free-swimming (Fuiman and Webb, 1988; Müller et al., 2000; Buss and Drapeau, 2001). Of these, only Buss and Drapeau (2001) quantitatively compared free-swimming and fictive locomotor patterns and did so just at $4 \mathrm{dpf}$, reporting a free-swimming and fictive correspondence that our study corroborates. Expanding on this, we found a strong correspondence between free-swimming and fictive locomotor patterns in the developmental switch from long to short swimming episodes between 3 and $4 \mathrm{dpf}$ (Fig. 1). Furthermore, there was correspondence between the altered free-swimming and fictive locomotor patterns resulting from blocking specific DARs (Figs. 2, 3 ) or from chemogenetic ablation of $o t p b$ cells (Fig. 7). Interestingly, behavioral and morphological factors in the free-swimming assay that could plausibly influence the locomotor pattern are not present 
A

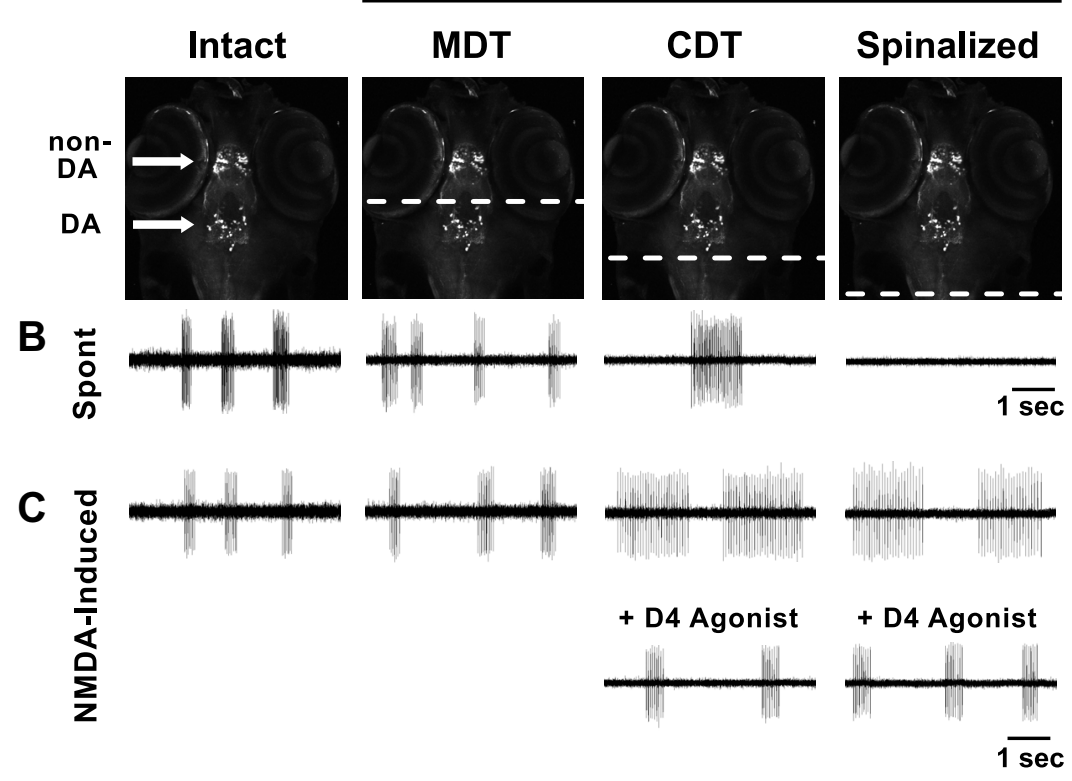

D

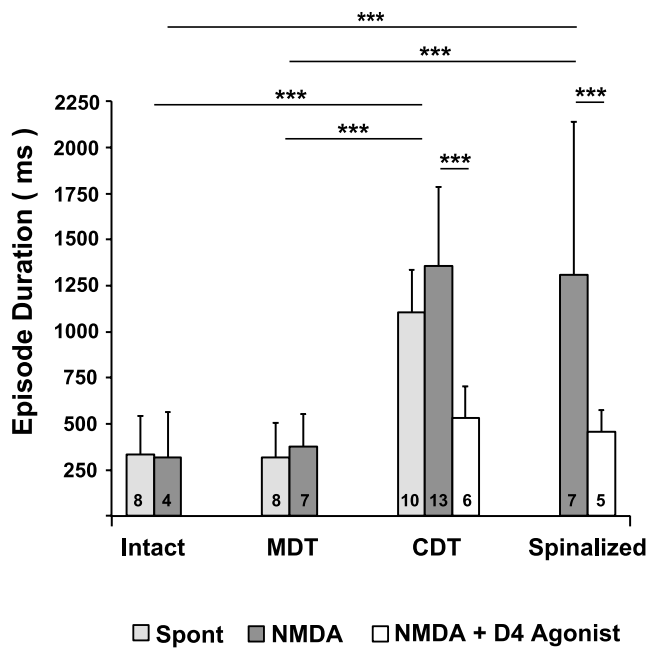

Figure 8. Removal of the caudal diencephalon reverts the mature locomotor pattern to an immature pattern that is rescued by $D_{4} R$ agonism. $A$, Schematic of demarcated transections, denoted by dashed white lines, with respect to the rostral non-DAergic and caudal DAergic otpb ${ }^{+}$populations in $\operatorname{Tg}(\text { otpb.A:nfs } B \text { - egfp })^{\text {zc77 }}$ larvae. Repeated image is from a confocal $z$-stack projection with a ventral view in whole-mount embryos; rostral is to the top. $B, C$, Voltage traces from extracellular peripheral nerve recordings showing fictive swimming of $4-7 \mathrm{dpf}$ larvae that is spontaneous in $\boldsymbol{B}$ and NMDA-induced in C. D. Mean fictive swimming episode durations (milliseconds) for spontaneous and NMDA-induced activity in intact, MDT, CDT, and spinalized-transected preparations. Numbers at the base of bars in bar graphs denote the sample sizes of fish for each experimental group. ${ }^{* *} p<0.001$.

in the immobilized fictive preparation. These factors include the following: (1) group interactions, because our assay tracked multiple larvae in the same arena; and (2) the absence or presence of an inflated swim bladder, which dramatically affect buoyancy and resultant free-swimming trajectories (Lindsey et al., 2010). Hence, the strong correspondence between the free-swimming and fictive locomotor patterns under all of our experimental conditions demonstrates that behavioral and morphological factors are not responsible for the developmental switch from the immature to mature episodic locomotor pattern, suggesting that the mechanisms underlying this switch are intrinsic to the nervous system.

\section{Role for spinal $\mathrm{D}_{4} \mathrm{R}$ signaling in sculpting mature locomotor pattern}

We found that specifically $\mathrm{D}_{4} \mathrm{R}$ signaling in the spinal cord is required for the advent and maintenance of the mature episodic locomotor pattern. Conversely, we demonstrate that all DAR subtypes play a role in influencing the amount of free-swimming activity. The specific DAR antagonists that we administered elicited unique locomotor phenotypes (Figs. 2, 3), indicating that each drug concentration was sufficient to differentially modulate behavior. The $\mathrm{D}_{4} \mathrm{R}$ antagonist group was hypoactive, which is consistent with other studies (Giacomini et al., 2006; Boehmler et al., 2007), and exhibited the immature pattern of long swimming episodes. The $\mathrm{D}_{1} \mathrm{R}$ antagonist group was also hypoactive whereas the $\mathrm{D}_{2} / \mathrm{D}_{3} \mathrm{R}$ antagonist group was hyperactive, which is consistent with evidence for such opposing roles of these receptors (Souza et al., 2011), yet both of these groups exhibited the mature pattern of short swimming episodes. Therefore, we demonstrate that the amount of swimming activity is separable from the locomotor pattern during swimming activity, suggesting that non-overlapping neural mechanisms exist for these two phenomena. This interpretation is supported by evidence that, at $3 \mathrm{dpf}$, DAergic signaling in the brain influences the amount of swimming activity but not the locomotor pattern (Thirumalai and
Cline, 2008), which also suggests that our results of systemic DARmediated changes in the amount of swimming activity may be attributable to blocking DARs in the brain.

We elucidated that the site of action of systemic $\mathrm{D}_{4} \mathrm{R}$-mediated changes in the locomotor pattern is likely in the spinal cord, because spinalization of 4-7 dpf larvae reverted the fictive locomotor pattern to immature-like long swimming episodes (Fig. 4) that were transformed to mature-like short episodes via spinal $\mathrm{D}_{4} \mathrm{R}$ agonism (Fig. 5 ). The ability of $\mathrm{D}_{4} \mathrm{R}$ antagonism to both prevent and reverse the developmental switch in the locomotor pattern suggests that the $\mathrm{D}_{4} \mathrm{R}$-dependent expression of short swimming episodes is not attributable to $\mathrm{D}_{4} \mathrm{R}$ signaling simply providing a transient role in transforming neural circuit function. Rather, it indicates a requirement for ongoing $\mathrm{D}_{4} \mathrm{R}$-mediated maintenance of the swimming pattern, possibly in the form of providing neuromodulatory tone (Hauber, 2010) to spinal locomotor circuits.

Our functional evidence for spinal $\mathrm{D}_{4} \mathrm{R}$ signaling is supported by previous in situ hybridization studies of DAR mRNA expression patterns and suggests a distinctive functional role for a specific $\mathrm{D}_{4} \mathrm{R}$ subtype. Among $\mathrm{D}_{2}$-like DARs, three $\mathrm{D}_{2} \mathrm{Rs}$ (drd2a,b,c), one $\mathrm{D}_{3} \mathrm{R}$ (drd3), and three $\mathrm{D}_{4} \mathrm{Rs}$ (drd4a,b,c) have been identified in zebrafish larvae. Interestingly, all of these DAR mRNAs are expressed in the zebrafish spinal cord by $2 \mathrm{dpf}$ (Boehmler et al., 2004, 2007) except drd4b, which is present at $5 \mathrm{dpf}$ (Boehmler et al., 2007). Because these studies did not examine expression patterns at 3 and $4 \mathrm{dpf}$, it is possible that the advent of spinal drd $4 \mathrm{~b}$ expression correlates with, and may underlie, the developmental switch in the locomotor pattern between 3 and $4 \mathrm{dpf}$.

Role for DAergic otpb population, via the DDT, in driving spinal $\mathrm{D}_{4} \mathrm{R}$-mediated sculpting of mature locomotor pattern Among the diencephalic DAergic populations in zebrafish (DC1DC6), the otp populations comprise four of the six (DC2, DC4, DC5, and DC6) (Kastenhuber et al., 2010). Of these otpb popu- 
lations, three of four (DC2, DC4, and DC5) exclusively comprise the DDT (Tay et al., 2011), and we hypothesized that some or all of these populations were involved in the spinal DAergicmediated shortening of swimming episodes because the DDT provides the exclusive source of DA to the spinal cord (Kastenhuber et al., 2010). Because the $\operatorname{Tg}(o t p b . A: n f s B-e g f p)^{\text {zc } 77}$ line that we used (Figs. 6-8) drives expression in the majority of DC4, DC5, and DC6 populations (Fujimoto et al., 2011), we were able to selectively chemogenetically ablate two of the three (DC4 and DC5) otp neuronal populations that comprise the DDT. Because this chemogenetic ablation was sufficient to perpetuate the immature locomotor pattern, it suggests that DC4 and/or DC5 DAergic otp $b$ populations are necessary for the developmental switch to short swimming episodes.

Conversely, we demonstrated that the non-DAergic otp $b$ population is not necessary to confer the mature episodic locomotor pattern, which persisted after removal of these cells by way of mid-diencephalic transections (Fig. 8). Importantly, this transection also removed the majority of DAergic non-otp $b$ populations, including a robust local subpallial DAergic system (Tay et al., 2011), as well as ascending subpallial projections of the DAergic $o t p b$ population (Tay et al., 2011), suggesting that these components are also not necessary for the expression of the mature episodic locomotor pattern. Removal of the DAergic otpb population by transecting at the caudal diencephalon reestablished the immature locomotor pattern (Fig. 8), demonstrating that the caudal diencephalon, which distinguishes MDT from CDT preparations, plays a role in conferring the expression of the mature locomotor pattern. Because the hindbrain remains intact in CDT preparations and these larvae are unable to maintain short swimming episodes, it suggests that serotonergic and noradrenergic projections into the spinal cord, which exclusively originate in the hindbrain (McLean and Fetcho, 2004b; Kastenhuber et al., 2010), are not sufficient for or do not contribute to the mature pattern of short swimming episodes. This interpretation is consistent with a previous study that showed that serotonin reduces the quiescent periods between swimming episodes but does not influence the active properties of swimming episodes themselves, including episode duration (Brustein et al., 2003). Although the literature is lacking on any role for noradrenaline on the pattern of swimming episodes, the number of noradrenergic axonal projections to the spinal cord of zebrafish larvae is markedly small compared with that of DAergic axonal projections via the DDT, as demonstrated by an essentially unaffected descending catecholaminergic tract after genetic knockdown of the noradrenaline system (Kastenhuber et al., 2010).

Although many cell populations and fibers of passage are contained within the caudal diencephalon, the physical removal of this region resulted in a similar locomotor phenotype as that induced from selective chemogenetic ablation of otp $b$ cells, suggesting that DAergic otp $b$ cells are the critical caudal diencephalic component. Consistent with this conclusion, in the absence of the caudal diencephalon, the reestablished immature locomotor pattern was rescued to a mature-like pattern via $\mathrm{D}_{4} \mathrm{R}$ agonism (Fig. 8). Moreover, the equitable efficacy of $\mathrm{D}_{4} \mathrm{R}$ agonism to rescue the locomotor pattern in CDT and spinalized preparations (Fig. 8) indicates the sufficiency of spinal $\mathrm{D}_{4} \mathrm{R}$ signaling to confer the mature episodic locomotor pattern. This provides additional credence to the conclusion that caudal diencephalic DAergic otpb cells comprising the DDT act on $\mathrm{D}_{4}$ Rs in the spinal cord, rather than the brain, to confer the mature episodic locomotor pattern.

\section{Conserved functional role of the vertebrate DDT}

This study is the first to elucidate the role of the DDT in a developing vertebrate in vivo. The conserved transcriptional identity (Ryu et al., 2007), somatic location, morphology, and projection pattern (Takada et al., 1988; Tay et al., 2011) of the DAergic otp population and DDT suggest that it has a conserved locomotor function across vertebrates. In support of this rationale in developing mammals, all DAR mRNAs are expressed across the dorsoventral axis of the neonatal mouse spinal cord and specifically in motor neurons (Zhu et al., 2007), which are modulated by DA during fictive locomotion in vitro as early as postnatal day 2 (P2) (Han et al., 2007). Moreover, before neonatal rodents can support their body weight to start walking, they are capable of coordinated locomotion in the form of L-DOPA-induced air-stepping that is dependent on DAR signaling (Sickles et al., 1992; McCrea et al., 1997). Spinalized in vitro preparations have demonstrated that this DAR signaling acts at the level of the spinal cord (McEwen et al., 1997). This early DAR-dependent signaling in the spinal cord may play a developmental role, since L-DOPAinduced air-stepping develops from being bipedal, at $\mathrm{P} 0$, to quadrupedal, by P5 (Van Hartesveldt et al., 1991). In disease states, for example, hypoxic-ischemic injury to the developing brain, DAergic cell populations are at risk and may contribute to the impaired motor development after these injuries (Bax et al., 2006). Hence, the evolutionarily conserved anatomical, genetic, and in vitro evidence suggests that, similar to our findings in zebrafish, the mammalian DDT may also contribute to locomotor development.

\section{References}

Bax JJ, Poldermans D, Schuijf JD, Scholte AJ, Elhendy A, van der Wall EE (2006) Imaging to differentiate between ischemic and nonischemic cardiomyopathy. Heart Fail Clin 2:205-214.

Boehmler W, Obrecht-Pflumio S, Canfield V, Thisse C, Thisse B, Levenson R (2004) Evolution and expression of D2 and D3 dopamine receptor genes in zebrafish. Dev Dyn 230:481-493.

Boehmler W, Carr T, Thisse C, Thisse B, Canfield VA, Levenson R (2007) D4 dopamine receptor genes of zebrafish and effects of the antipsychotic clozapine on larval swimming behaviour. Genes Brain Behav 6:155-166.

Bonkowsky JL, Chien CB (2005) Molecular cloning and developmental expression of foxP2 in zebrafish. Dev Dyn 234:740-746.

Bonkowsky JL, Wang X, Fujimoto E, Lee JE, Chien CB, Dorsky RI (2008) Domain-specific regulation of foxP2 CNS expression by lef1. BMC Dev Biol 8:103.

Borla MA, Palecek B, Budick S, O’Malley DM (2002) Prey capture by larval zebrafish: evidence for fine axial motor control. Brain Behav Evol 60:207-229.

Branson K, Robie AA, Bender J, Perona P, Dickinson MH (2009) Highthroughput ethomics in large groups of Drosophila. Nat Methods $6: 451-457$

Brustein E, Chong M, Holmqvist B, Drapeau P (2003) Serotonin patterns locomotor network activity in the developing zebrafish by modulating quiescent periods. J Neurobiol 57:303-322.

Buss RR, Drapeau P (2001) Synaptic drive to motoneurons during fictive swimming in the developing zebrafish. J Neurophysiol 86:197-210.

Curado S, Stainier DY, Anderson RM (2008) Nitroreductase-mediated cell/ tissue ablation in zebrafish: a spatially and temporally controlled ablation method with applications in developmental and regeneration studies. Nat Protoc 3:948-954.

Farrell TC, Cario CL, Milanese C, Vogt A, Jeong JH, Burton EA (2011) Evaluation of spontaneous propulsive movement as a screening tool to detect rescue of Parkinsonism phenotypes in zebrafish models. Neurobiol Dis 44:9-18.

Fuiman LA, Webb PW (1988) Ontogeny of routine swimming activity and performance in zebra danios (Teleostei: Cyprinidae). Anim Behav 36:250-261.

Fujimoto E, Stevenson TJ, Chien CB, Bonkowsky JL (2011) Identification of 
a dopaminergic enhancer indicates complexity in vertebrate dopamine neuron phenotype specification. Dev Biol 352:393-404.

Giacomini NJ, Rose B, Kobayashi K, Guo S (2006) Antipsychotics produce locomotor impairment in larval zebrafish. Neurotoxicol Teratol 28:245-250.

Han P, Nakanishi ST, Tran MA, Whelan PJ (2007) Dopaminergic modulation of spinal neuronal excitability. J Neurosci 27:13192-13204.

Hauber W (2010) Dopamine release in the prefrontal cortex and striatum: temporal and behavioural aspects. Pharmacopsychiatry 43 [Suppl 1]:S32-S41.

Holzschuh J, Ryu S, Aberger F, Driever W (2001) Dopamine transporter expression distinguishes dopaminergic neurons from other catecholaminergic neurons in the developing zebrafish embryo. Mech Dev 101:237-243.

Kastenhuber E, Kratochwil CF, Ryu S, Schweitzer J, Driever W (2010) Genetic dissection of dopaminergic and noradrenergic contributions to catecholaminergic tracts in early larval zebrafish. J Comp Neurol 518:439-458.

Kokel D, Bryan J, Laggner C, White R, Cheung CY, Mateus R, Healey D, Kim S, Werdich AA, Haggarty SJ, Macrae CA, Shoichet B, Peterson RT (2010) Rapid behavior-based identification of neuroactive small molecules in the zebrafish. Nat Chem Biol 6:231-237.

Kwan KM, Fujimoto E, Grabher C, Mangum BD, Hardy ME, Campbell DS, Parant JM, Yost HJ, Kanki JP, Chien CB (2007) The Tol2kit: a multisite gateway-based construction kit for Tol2 transposon transgenesis constructs. Dev Dyn 236:3088-3099.

Lindsey BW, Smith FM, Croll RP (2010) From inflation to flotation: contribution of the swimbladder to whole-body density and swimming depth during development of the zebrafish (Danio rerio). Zebrafish 7:85-96.

Masino MA, Fetcho JR (2005) Fictive swimming motor patterns in wild type and mutant larval zebrafish. J Neurophysiol 93:3177-3188.

McCrea AE, Stehouwer DJ, Van Hartesveldt C (1997) Dopamine D1 and D2 antagonists block L-DOPA-induced air-stepping in decerebrate neonatal rats. Brain Res Dev Brain Res 100:130-132.

McDearmid JR, Drapeau P (2006) Rhythmic motor activity evoked by NMDA in the spinal zebrafish larva. J Neurophysiol 95:401-417.

McEwen ML, Van Hartesveldt C, Stehouwer DJ (1997) L-DOPA and quipazine elicit air-stepping in neonatal rats with spinal cord transections. Behav Neurosci 111:825-833.

McLean DL, Fetcho JR (2004a) Relationship of tyrosine hydroxylase and serotonin immunoreactivity to sensorimotor circuitry in larval zebrafish. J Comp Neurol 480:57-71.

McLean DL, Fetcho JR (2004b) Ontogeny and innervation patterns of dopaminergic, noradrenergic, and serotonergic neurons in larval zebrafish. J Comp Neurol 480:38-56.

Müller UK, Stamhuis EJ, Videler JJ (2000) Hydrodynamics of unsteady fish swimming and the effects of body size: comparing the flow fields of fish larvae and adults. J Exp Biol 203:193-206.

Paulus W, Dowling P, Rijsman R, Stiasny-Kolster K, Trenkwalder C, de Weerd A (2007) Pathophysiological concepts of restless legs syndrome. Mov Disord 22:1451-1456.

Pisharath H, Parsons MJ (2009) Nitroreductase-mediated cell ablation in transgenic zebrafish embryos. Methods Mol Biol 546:133-143.

Qu S, Ondo WG, Zhang X, Xie WJ, Pan TH, Le WD (2006) Projections of diencephalic dopamine neurons into the spinal cord in mice. Exp Brain Res 168:152-156.

Qu S, Le W, Zhang X, Xie W, Zhang A, Ondo WG (2007) Locomotion is increased in a11-lesioned mice with iron deprivation: a possible animal model for restless legs syndrome. J Neuropathol Exp Neurol 66:383-388.

Reiner A, Medina L, Veenman CL (1998) Structural and functional evolution of the basal ganglia in vertebrates. Brain Res Brain Res Rev 28:235-285.

Rihel J, Prober DA, Arvanites A, Lam K, Zimmerman S, Jang S, Haggarty SJ, Kokel D, Rubin LL, Peterson RT, Schier AF (2010) Zebrafish behavioral profiling links drugs to biological targets and rest/wake regulation. Science 327:348-351.

Ryu S, Mahler J, Acampora D, Holzschuh J, Erhardt S, Omodei D, Simeone A, Driever W (2007) Orthopedia homeodomain protein is essential for diencephalic dopaminergic neuron development. Curr Biol 17:873-880.

Sickles AE, Stehouwer DJ, Van Hartesveldt C (1992) Dopamine D1 and D2 antagonists block L-dopa-elicited air-stepping in neonatal rats. Brain Res Dev Brain Res 68:17-22.

Smeets WJ, González A (2000) Catecholamine systems in the brain of vertebrates: new perspectives through a comparative approach. Brain Res Brain Res Rev 33:308-379.

Souza BR, Romano-Silva MA, Tropepe V (2011) Dopamine $\mathrm{D}_{2}$ receptor activity modulates Akt signaling and alters GABAergic neuron development and motor behavior in zebrafish larvae. J Neurosci 31:5512-5525.

Straw AD, Dickinson MH (2009) Motmot, an open-source toolkit for realtime video acquisition and analysis. Source Code Biol Med 4:5.

Takada M, Li ZK, Hattori T (1988) Single thalamic dopaminergic neurons project to both the neocortex and spinal cord. Brain Res 455:346-352.

Taniguchi W, Nakatsuka T, Miyazaki N, Yamada H, Takeda D, Fujita T, Kumamoto E, Yoshida M (2011) In vivo patch-clamp analysis of dopaminergic antinociceptive actions on substantia gelatinosa neurons in the spinal cord. Pain 152:95-105.

Tay TL, Ronneberger O, Ryu S, Nitschke R, Driever W (2011) Comprehensive catecholaminergic projectome analysis reveals single-neuron integration of zebrafish ascending and descending dopaminergic systems. Nat Commun 2:171.

Thirumalai V, Cline HT (2008) Endogenous dopamine suppresses initiation of swimming in prefeeding zebrafish larvae. J Neurophysiol 100:1635-1648.

Tierney KB (2011) Behavioural assessments of neurotoxic effects and neurodegeneration in zebrafish. Biochim Biophys Acta 1812:381-389.

Van Hartesveldt C, Sickles AE, Porter JD, Stehouwer DJ (1991) L-dopainduced air-stepping in developing rats. Brain Res Dev Brain Res 58:251-255.

Zhao H, Zhu W, Pan T, Xie W, Zhang A, Ondo WG, Le W (2007) Spinal cord dopamine receptor expression and function in mice with 6-OHDA lesion of the A11 nucleus and dietary iron deprivation. J Neurosci Res 85:1065-1076.

Zhu H, Clemens S, Sawchuk M, Hochman S (2007) Expression and distribution of all dopamine receptor subtypes $(\mathrm{D}(1)-\mathrm{D}(5))$ in the mouse lumbar spinal cord: a real-time polymerase chain reaction and non-autoradiographic in situ hybridization study. Neuroscience 149:885-897. 